\title{
Tandem repeat regions within the Burkholderia pseudomallei genome and their application for high resolution genotyping
} Jana M U'Ren ${ }^{\dagger 1}$, James M Schupp ${ }^{\dagger 1}$, Talima Pearson ${ }^{1}$, Heidie Hornstra ${ }^{1}$, Christine L Clark Friedman ${ }^{1}$, Kimothy L Smith1, Rebecca R Leadem Daugherty', Shane D Rhoton1, Ben Leadem¹, Shalamar Georgia1, Michelle Cardon ${ }^{1}$, Lynn Y Huynh ${ }^{1}$, David DeShazer ${ }^{2}$, Steven P Harvey ${ }^{3}$, Richard Robison ${ }^{4}$, Daniel Gal ${ }^{5}$, Mark J Mayo ${ }^{5}$, David Wagner ${ }^{1}$, Bart J Currie ${ }^{5}$ and Paul Keim*1

Address: ${ }^{1}$ Northern Arizona University, Center for Microbial Genetics and Genomics, Box 5640, Flagstaff Arizona, 86011 USA, ${ }^{2}$ U.S. Army Medical Research Institute for Infectious Diseases, Fort Detrick, Maryland 21702-5011 USA, ${ }^{3}$ U.S. Army Edgewood Chemical Biological Center, Aberdeen Proving Ground, Maryland 21010-5454 USA, ${ }^{4}$ Brigham Young University, Provo, Utah 84602 USA and ${ }^{5}$ Menzies School of Health Research, Charles Darwin University, Darwin, Northern Territory, Australia

Email: Jana M U'Ren - juren@email.arizona.edu; James M Schupp - James.Schupp@nau.edu; Talima Pearson - Talima.Pearson@nau.edu; Heidie Hornstra - Heidie.Hornstra-ONeill@NAU.EDU; Christine L Clark Friedman - Christine.Clark@nau.edu;

Kimothy L Smith - Kimothy.Smith@dhs.gov; Rebecca R Leadem Daugherty - r-leadem@northwestern.edu;

Shane D Rhoton - sdry78@umkc.edu; Ben Leadem - brl28@dana.ucc.nau.edu; Shalamar Georgia - smg63@dana.ucc.nau.edu;

Michelle Cardon - mlc@janetoriolesupply.com; Lynn Y Huynh - lyhuynh@emory.edu; David DeShazer - david.deshazer@amedd.army.mil; Steven P Harvey - steve.harvey@us.army.mil; Richard Robison - Richard_Robison@byu.edu; Daniel Gal - Daniel.Gal@menzies.edu.au; Mark J Mayo - Mark.Mayo@menzies.edu.au; David Wagner - David.Wagner@NAU.EDU; Bart J Currie - Bart.Currie@menzies.edu.au;

Paul Keim* - Paul.Keim@nau.edu

* Corresponding author †Equal contributors

Published: 30 March 2007

BMC Microbiology 2007, 7:23 doi:10.1 186/147/-2180-7-23
Received: 10 November 2006

Accepted: 30 March 2007

This article is available from: http://www.biomedcentral.com/I47I-2/80/7/23

(C) 2007 U'Ren et al; licensee BioMed Central Ltd.

This is an Open Access article distributed under the terms of the Creative Commons Attribution License (http://creativecommons.org/licenses/by/2.0), which permits unrestricted use, distribution, and reproduction in any medium, provided the original work is properly cited.

\begin{abstract}
Background: The facultative, intracellular bacterium Burkholderia pseudomallei is the causative agent of melioidosis, a serious infectious disease of humans and animals. We identified and categorized tandem repeat arrays and their distribution throughout the genome of B. pseudomallei strain K96243 in order to develop a genetic typing method for B. pseudomallei. We then screened 104 of the potentially polymorphic loci across a diverse panel of $3 \mathrm{I}$ isolates including $B$. pseudomallei, B. mallei and B. thailandensis in order to identify loci with varying degrees of polymorphism. A subset of these tandem repeat arrays were subsequently developed into a multiple-locus VNTR analysis to examine 66 B. pseudomallei and $21 \mathrm{~B}$. mallei isolates from around the world, as well as 95 lineages from a serial transfer experiment encompassing $\sim 18,000$ generations.

Results: B. pseudomallei contains a preponderance of tandem repeat loci throughout its genome, many of which are duplicated elsewhere in the genome. The majority of these loci are composed of repeat motif lengths of 6 to 9 bp with 4 to 10 repeat units and are predominately located in intergenic regions of the genome. Across geographically diverse $B$. pseudomallei and B.mallei isolates, the 32 VNTR loci displayed between 7 and 28 alleles, with Nei's diversity values ranging from 0.47 and 0.94 . Mutation rates for these loci are comparable $\left(>10^{-5}\right.$ per locus per generation) to that of the most diverse tandemly repeated regions found in other less diverse bacteria.
\end{abstract}


Conclusion: The frequency, location and duplicate nature of tandemly repeated regions within the $B$. pseudomallei genome indicate that these tandem repeat regions may play a role in generating and maintaining adaptive genomic variation. Multiple-locus VNTR analysis revealed extensive diversity within the global isolate set containing $B$. pseudomallei and $B$. mallei, and it detected genotypic differences within clonal lineages of both species that were identical using previous typing methods. Given the health threat to humans and livestock and the potential for B. pseudomallei to be released intentionally, MLVA could prove to be an important tool for fine-scale epidemiological or forensic tracking of this increasingly important environmental pathogen.

\section{Background}

The environmental saprophyte Burkholderia pseudomallei is the causative agent of melioidosis, a disease endemic to tropical regions of Southeast Asia and northern Australia. Symptoms range in severity from fatal sepsis and acute community-acquired pneumonia to benign and localized abscesses. Infection in humans and animals generally occurs through direct contact of open wounds or abrasions with contaminated water and soil, by ingestion of contaminated drinking water, or inhalation of infectious aerosols. Melioidosis is a serious public health threat in Thailand and northern Australia, where it is associated with a case fatality rate of approximately 50 and $20 \%$, respectively [1]. In addition, $B$. pseudomallei has recently attracted attention as a potential biological weapon, and is listed as a Category B biothreat agent by the U.S. Centers for Disease Control and Prevention (CDC) [2].

The close genetic relationship of B. pseudomallei to B. mallei has previously been demonstrated by DNA hybridization studies [3]. More recently, studies have revealed that B. mallei is a clonal lineage of B. pseudomallei, and its recent evolutionary divergence is marked by gene deletions and intra-chromosomal rearrangements [4-7]. B. mallei, the etiologic agent of glanders, is an obligate parasite of the family Equidae, but can also infect humans through direct contact with infected animals [8] or occupational exposure [9]. Glanders was once a globally distributed disease, but is currently predominant only in the Middle East, Africa, Asia and Central and South America. Due to its highly infectious nature and ability to infect via aerosol, it was used as a biological weapon during World War I and World War II $[10,11]$. It is also listed as a Category B biothreat agent by the CDC [2].

Due to the severe nature of melioidosis, the molecular epidemiology of $B$. pseudomallei has been investigated using various DNA restriction-based methods, including Pulse Field Gel Electorphoresis (PFGE) [12,13] and ribotyping $[14,15]$. PFGE has the ability to resolve potentially polymorphic, large DNA restriction fragments, while ribotyping uses restriction fragment length polymorphisms associated with rRNA genes [16]. Although both of these methods have been successful in the epidemiological tracking of pathogens [17], their technical nature can make large datasets more difficult to handle. Also, neither method is easily standardized for transfer throughout the scientific and public health community, and can often lack discriminatory power among closely related isolates within a species or between closely related species [18].

Other procedures that have been used for molecular typing of B. pseudomallei involve PCR, such as random amplified polymorphic DNA (RAPD) $[19,20]$ and multilocus sequence typing (MLST) [6]. RAPD detects differences in genomes by amplifying segments of unknown DNA. Drawbacks to this technique include the presence/ absence binary nature of the data and the difficulty in reproducing banding patterns between reactions (attributed to PCR artifacts). MLST uses concatenated nucleotide sequences from seven housekeeping genes, that are assumed to be selectively neutral or under purifying selection [21]. This method provides nucleotide data for multiple haplotypes, is easily amenable to phylogenetic analyses and can be standardized across laboratories. The MLST scheme developed for B. pseudomallei is also applicable to B. mallei and B. thailandensis. However, MLST can be time consuming and expensive, and most importantly lacks discriminatory power within closely related $B$. pseudomallei isolates and among the vast majority of $B$. malle $i$ isolates, which are all close genetic relatives [6].

Recently, a reliable PCR-based method using variablenumber tandem repeat (VNTR) loci has become a popular tool for the molecular typing of pathogens [18,22-25]. A VNTR locus consists of tandemly repeated sequences of DNA that vary in copy number, creating PCR amplicon size polymorphisms that are easily detected with gel electrophoresis. Due to increased mutation rates when compared to other regions of DNA and their multi-allelic nature, VNTRs allow superior discrimination between closely related isolates. These loci have been successfully implemented for forensic, epidemiological and phylogenetic analyses of bacterial pathogens with low genetic diversity, such as Bacillus anthracis, F. tularensis, and Y. pestis [23,26-30].

Due to the success of VNTR typing in other pathogens, the primary objective of this study was to develop a high-res- 
olution VNTR typing system for B. pseudomallei that is suitable for epidemiological, forensic, phylogenetic and population genetic studies. Thus the first task for this study was to characterize tandem repeat loci, including their distribution and frequency within the $B$. pseudomallei genome. Additionally, in order to develop a comprehensive multiple-locus VNTR typing system that utilizes loci with varying degrees of polymorphism, the second task was to screen loci that were characteristic of the tandem repeat loci throughout the genome and examine levels of polymorphism. Finally, in order to understand the effects that mechanisms such as recombination and mutation have on generating the high levels of diversity observed in this pathogen, it was essential to examine the mutation rates for the non-duplicated VNTR loci chosen for the typing system, as well as a representative sample of the duplicated tandem repeat regions. Furthermore, the estimation of mutation rates will allow for future epidemiological studies that model the transmission of melioidosis in natural populations, similar to published studies on plague [26].

In this manuscript we describe a multiple-locus VNTR analysis (MLVA) genotyping system in which 32 independent, tandemly inserted repeated motifs identified in the B. pseudomallei K96243 genome are amplified using fluorescently labeled primers in multiplexed PCRs and separated using capillary electrophoresis. These loci were highly polymorphic across a globally distributed set of 66 B. pseudomallei and $21 \mathrm{~B}$. mallei isolates, as well as a few very closely related $B$. pseudomallei isolates from an outbreak event and two individual patients.

\section{Results \\ Tandem repeats within the Burkholderia pseudomallei genome}

We observed that in comparison to other bacterial pathogens with similarly sized genomes, such as Bacillus anthracis Ames and Yersinia pestis CO92, the Burkholderia pseudomallei K96243 genome harbors a relatively large number of tandem repeat arrays (Figure 1). The large $(4,074,542 \mathrm{bp})$ chromosome of $B$. pseudomallei contains 285 (69.9 arrays/Mbp) while the small $(3,173,005 \mathrm{bp})$ chromosome contains 324 (102.1 arrays/Mbp) tandem repeat arrays (Table 1 ). In contrast, the $Y$. pestis genome contains only 174 arrays and B. anthracis contains just 66 arrays, at densities of 37.4 arrays/Mb interval and 12.6 arrays/Mb, respectively. In $B$. pseudomallei, tandem repeat motif sizes on both chromosomes ranged from 3 to $16 \mathrm{bp}$ with copy numbers ranging from 4 to 21 units (Figure 2, A1 and A2). Non-triplet repeat motifs were more common in intragenic regions than inside genes (Figure 2, B1 and B2).

\section{Distribution and location of tandem repeats}

A $\chi^{2}$ goodness-of-fit test of the "observed" B. pseudomallei tandem repeat distribution to an "expected" Poisson distribution was significant for both the large $(\mathrm{p}<0.001)$ and small chromosomes $(\mathrm{p}<0.001)$ using $10 \mathrm{~Kb}$ intervals (Figure 3). The non-random observed distributions for both chromosomes are consistent with a clustered arrangement of arrays throughout both chromosomes. Additionally, the majority of the tandem repeats were found in intergenic regions of the chromosomes: $74.7 \%$ $(\mathrm{n}=213)$ tandem repeats on the large chromosome and $68.2 \%(\mathrm{n}=221)$ on the small chromosome. However, a portion of these arrays $(28.1 \%$ on the large chromosome and $35.2 \%$ on the small chromosome) were found inside or within 40 base pairs upstream of predicted ORFs (Table 1 ). Longer arrays ( $\geq 11$ repeat units), including even those with triplet motifs, tended not to be found inside predicted protein coding regions on the large chromosome (Figure 2A1). Conversely, on the small chromosome, longer arrays with triplet repeat motifs were found in both inter- and intragenic locations in almost equal numbers (Figure 2A2). It was also observed that four-fold more degenerate arrays were found on the small chromosome than on the large, and the majority of these degenerate arrays were located inside coding regions (Figure 2A1, and 2A2).

We found that $36.3 \%$ of the total number of tandem repeat arrays on both chromosomes of $B$. pseudomallei are duplicated, at least partially ( $\geq 20$ bp and $\geq 80 \%$ similarity), in other locations on either chromosome (Table 1). Most of these duplications were found in intergenic regions of the chromosomes and involved the repeat motif only and not the flanking sequences. The majority of duplicated tandem repeats on the large chromosome were, in fact, duplicated on the small chromosome, rather than on the large chromosome. In contrast, arrays duplicated on the small chromosome were found in equal numbers on both chromosomes (Table 1). Additionally, total array lengths were typically longer for duplicated tandem arrays. For example, 104 of the 108 duplicated arrays on the large chromosome, and 112 of the 114 duplicated arrays on the small chromosomes are larger than $200 \mathrm{bp}$, with the largest almost $6000 \mathrm{bp}$ in size. It was observed that repeat regions that contained more than 20 repeat copies were found to be duplicated in some fashion, and repeat motifs of six and seven bp were more often duplicated than not (Figure 2).

\section{MLVA development}

In order to develop a MLVA system for B. pseudomallei, a variety of array sizes were screened, from 2 bp repeat motif by 7 repeat copy unit (i.e. $2 \times 7$ ) to degenerate repeat arrays greater than $500 \mathrm{bp}$ but less than $1000 \mathrm{bp}$, for a total of 104 VNTR loci. We also screened both intra- and 
Table I: Summary of B. pseudomallei chromosomal repeat region frequency, duplication and location in coding regions

\begin{tabular}{|c|c|c|c|c|c|c|c|c|c|c|c|}
\hline Chromosome & Size (bp) & GC\% & $\begin{array}{c}\text { Tandem } \\
\text { repeat } \\
\text { (TR) } \\
\text { regionsł }\end{array}$ & TR/IO kb & $\begin{array}{c}\text { \#TR's in } \\
\text { Sanger } \\
\text { CDS }\end{array}$ & $\begin{array}{c}\text { \#TR's } \\
\text { within } 40 \\
\text { bp 5' of } \\
\text { Sanger } \\
\text { CDS }\end{array}$ & $\begin{array}{c}\text { \#TR's } \\
\text { within } 40 \\
\text { bp 3' of } \\
\text { Sanger } \\
\text { CDS }\end{array}$ & $\begin{array}{c}\text { cis only } \\
\text { duplicate } \\
\text { d TRs } \\
\text { (in CDS)* }\end{array}$ & $\begin{array}{c}\text { trans only } \\
\text { duplicate } \\
\text { d TRs } \\
\text { (in CDS)* }\end{array}$ & $\begin{array}{l}\text { Both cis } \\
\text { and trans } \\
\text { duplicated } \\
\text { TRs (in } \\
\text { CDS)* }\end{array}$ & $\begin{array}{l}\text { total dup } \\
\text { arrays } \\
\text { (in CDS)* }\end{array}$ \\
\hline Large & $4,074,542$ & 67.7 & 285 & 0.699 & $72 * *$ & 8 & 43 & $22(4)$ & $56(12)$ & $30(2)$ & $108(18)^{* * * *}$ \\
\hline Small & $3,173,005$ & 68.5 & 324 & 1.021 & $103 * *$ & 11 & 42 & $25(8)$ & $48(14)$ & 41 (13) & $114(35)^{* * * *}$ \\
\hline Total & $7,247,547$ & $68.1+$ & 609 & $0.860^{\dagger}$ & $175^{* *}$ & 19 & 85 & $47(12)$ & $104(26)$ & $7 I(15)$ & $222(53)^{* * * * * *}$ \\
\hline
\end{tabular}

$\ddagger$ Regions with repeats $\geq 2$ bp, $\geq 4$ repeat units and array sizes $\geq 30$ bp

$\dagger$ Average number

*duplications $\geq 20$ bp and $80 \%$ similarity

**all but 4 and 8 (XI and X2, resp.) of the non degenerate arrays had RU sizes of 3 bp multiples

$* * *$ Average duplication size of $50 \mathrm{bp}$

intergenically located arrays. Criteria used for including loci in the MLVA system were 1) variation within the screening panel (see Methods), either within the globally distributed or locally distributed outbreak sets, 2) robust (> 80\% success) PCR amplification, and 3 ) highly discrete PCR amplicon sizes (minimal partial repeat differences), based upon locus repeat unit motif. Thirty-two loci met the above three criteria and were chosen for MLVA development (Tables 2 and 3).

\section{B. pseudomallei and B. mallei genetic relationships}

The 32-locus MLVA system was used to characterize $66 \mathrm{~B}$. pseudomallei and $21 \mathrm{~B}$. mallei isolates from diverse geographic locations (Table 4). These loci provide high levels of discrimination among different isolates of B. pseudoma$l l e i$, with the number of alleles ranging between 7 to 28 , and Nei's diversity values between 0.47 and 0.94 across all $B$. pseudomallei and B. mallei isolates (Table 3). Furthermore, the MLVA loci amplified equally well in both $B$. pseudomallei and closely related B. mallei strains, and showed variation between and among the two closely related species. MLVA loci did not PCR amplify in the more genetically distant $B$. thailandensis and B. cepacia.

Analysis of allelic variation at 23 loci using a Neighbor Joining distance algorithm revealed 62 genotypes among the $66 \mathrm{~B}$. pseudomallei isolates and 19 genotypes among the $21 \mathrm{~B}$. mallei isolates. Phylogenetic analysis of these VNTR data provided an extremely high level of strain discrimination even within $B$. pseudomallei isolates from single melioidosis patients (Patient 465 and chronic lung patient) and within isolates from a single $B$. pseudomallei outbreak focus in Australia (Goat Farms 1 and 2) (Figure 4). The average pairwise genetic distance was 0.86 for $B$. pseudomallei, and 0.61 for B. mallei.

A phylogram depicting this analysis indicates four highly diverse major clusters among the two Burkholderia sp., although there is less than $50 \%$ bootstrap support for these branches (Figure 4). These major clusters did not reveal any noticeable geographic or temporal relation- ships, with isolates from the same country or the same time period occurring in all groups. However, there are many instances in which the relationships between closely related isolates demonstrate clear geographic correlations with solid statistical support (Figure 4). Additionally, the tree indicates that overall, B. pseudomallei is much more diverse than $B$. mallei, although this could be due to the less geographically diverse nature of the $B$. mal$l e i$ isolates. The tree clearly shows that the $B$. mallei isolates form a monophyletic group derived from a $B$. pseudomallei ancestor. The split between $B$. mallei and $B$. pseudomallei is supported by two MLVA loci (3564 k and $2445 \mathrm{k}$ ) that contain multiple alleles specific to $B$. mallei.

A comparison of a subset of isolates to other typing methods revealed that MLVA is much more discriminating between closely related isolates. MLST data for 37 of the $66 \mathrm{~B}$. pseudomallei and four of the $21 \mathrm{~B}$. mallei isolates used in this study were obtained from the online database [31]. A comparison of MLST and MLVA for these $37 \mathrm{~B}$. pseudomallei isolates revealed seven instances where MLST sequence types were identical between isolates, while MLVA genotypes were different in all but two of these instances (Figure 4). Of particular note was the single MLST genotype for $B$. mallei and the multiple MLVA genotypes for the same isolates $(n=4)$. Additionally, a ribotyping study revealed three genotypes for seven of the B. mallei isolates (T2, T4, T5, T7, T9, GB5, GB6), while MLVA identified unique genotypes for every isolate [32].

\section{Mutation rates of tandem repeats}

Parallel serial passages experiments (PSPE) from a single $B$. pseudomallei isolate resulted in estimated $\sim 18,000$ generations of growth from which lineages were analyzed for variation in all MLVA loci. Mutational events were observed in 12 VNTR loci; the number and type of mutations observed are shown in Table 5. We observed comparable numbers of mutations for loci on each chromosome. There was a noticeable trend towards single repeat mutations $(\mathrm{p}=0.0001)$ as well as bias towards insertion mutations $(\mathrm{p}=0.0736)$ (Table 5). No discerna- 


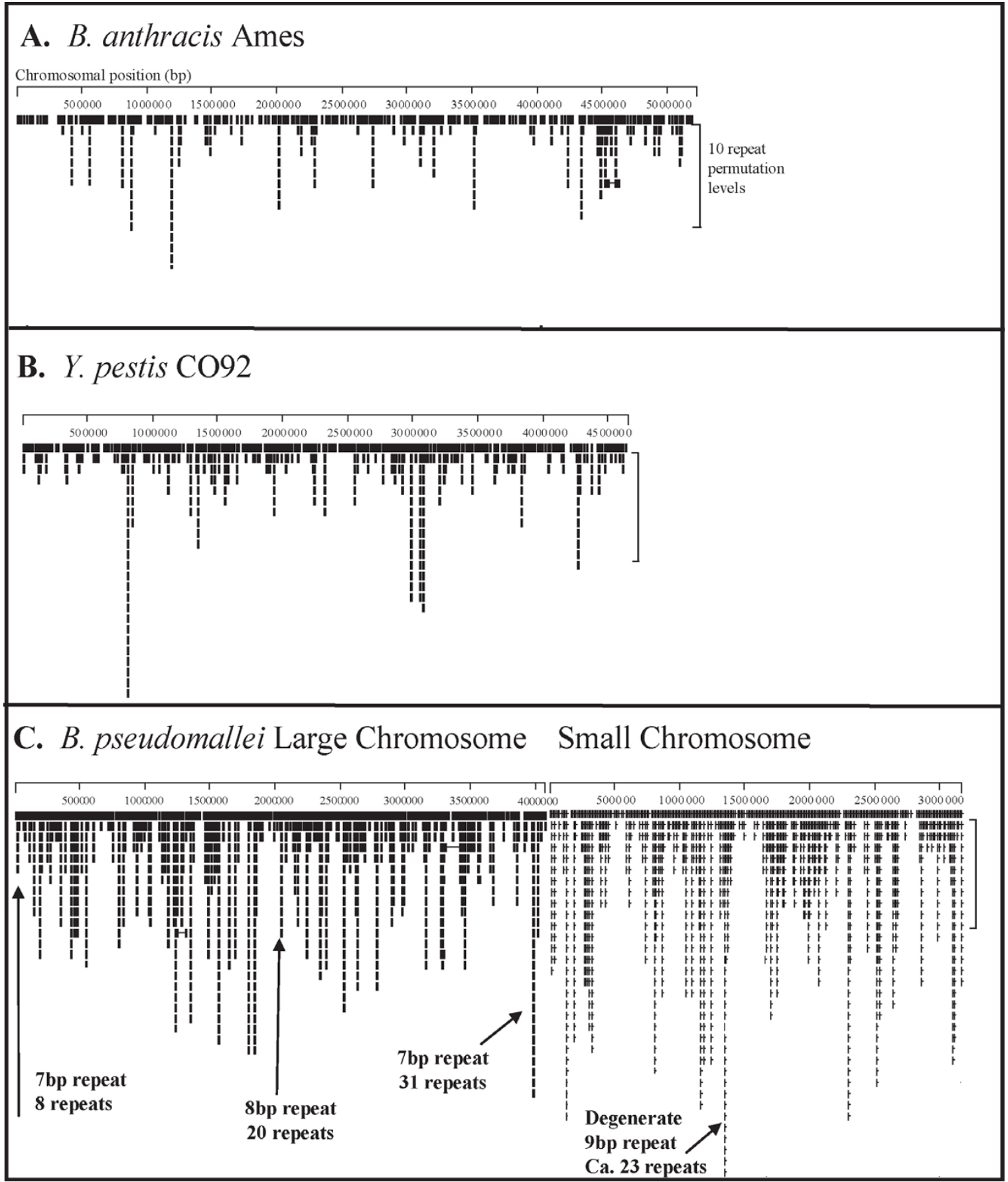

Figure I

Linear repeat array distribution of B. anthracis, $Y$. pestis and B. pseudomallei chromosomes. Nucleic acid repeat region "icicle" plots were generated with DNAStar GeneQuest software (Madison, WI). The horizontal scale indicates the linear position in base pairs along the respective chromosomes from the start position of the GenBank FASTA file sequence. The scale bar to the right of each icicle plot indicates 10 possible repeat sequence combinations as found by the GeneQuest software. The overall length, or number of possible repeat combinations of each icicle, is a measure of the size of the repeated sequence array found at that position. In general, the longer the icicle, the larger the repeat array. Note that both perfect and degenerate repeat arrays are found and displayed by GeneQuest, as indicated by the arrows and notes in panel $C$. The number of arrays/Mbp and total arrays are all repeat regions found by the software package Tandem Repeats Finder larger than 30 bp and with an internal similarity greater than or equal to $80 \%$. 


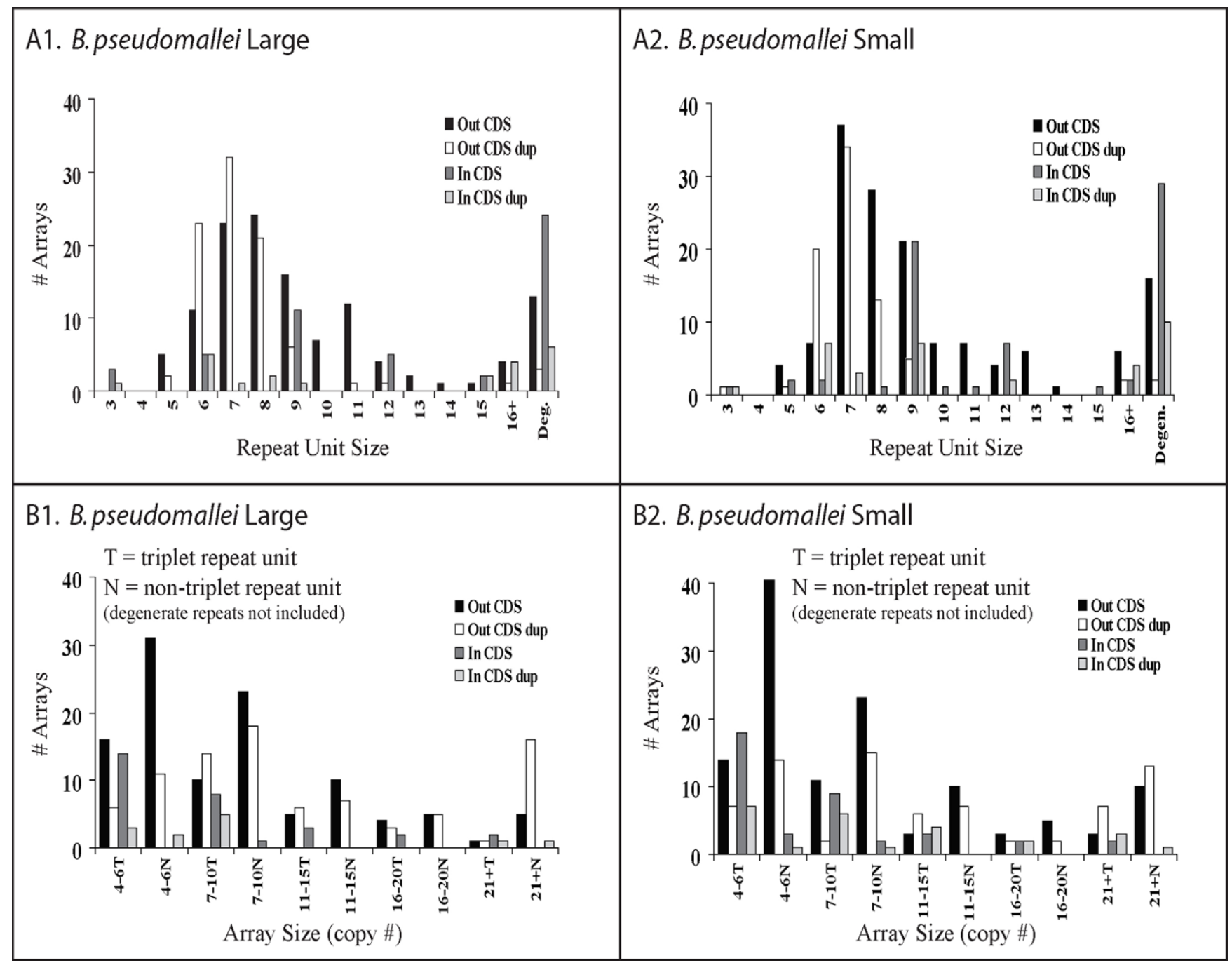

Figure 2

Repeat region motif size and total array size distribution. A) Frequencies of arrays consisting of different size repeat motifs in inter-, intragenic and duplicated locations. Degenerate repeats were determined as described in the Materials and Methods Section. B) Frequencies of arrays consisting of different total size classes, again in inter-, intragenic and duplicated locations, based upon triplet and non-triplet repeat motif copy number. Degenerate arrays are not included as consensus repeat motifs were not determined.

ble pattern was observed between loci that had mutations and those without mutations with respect to array size, repeat motif GC \%, and/or amplification characteristics. The number of successful lineage PCR amplifications for the mutating MLVA loci ranged from 75-95 (out of 95 possible), averaging $90.25 \pm 5.7$; while those from the non-mutating loci ranged from 82-95, averaging $92.25 \pm$ 3.1 (data not shown). (The basis of these failures is under investigation, but all mutation rates were corrected appropriately for these missing data.) We observed an average of 1.67 mutations per locus, and mutation rates for individual loci ranged from $5.3 \times 10^{-5}$ to $1.7 \times 10^{-4}$. The combined mutation rate across all 32 loci was $1.113 \times 10^{-3}$, which represents a discrimination power estimator for this MLVA typing system (Table 5). It is similar to the Y. pestis MLVA system rate and greater than the E. coli rate.

We also examined mutation rates for 17 tandem repeat loci, not included in the final MLVA system, containing arrays found to be duplicated in up to four different locations within and/or between chromosomes (Table 6). In contrast to the MLVA loci, all duplicated loci screened consisted of either six or seven bp repeat motifs, as these were most commonly found with larger duplicated regions in the K96243 strain. Also, while the number of mutations for the duplicated arrays was equal to the 


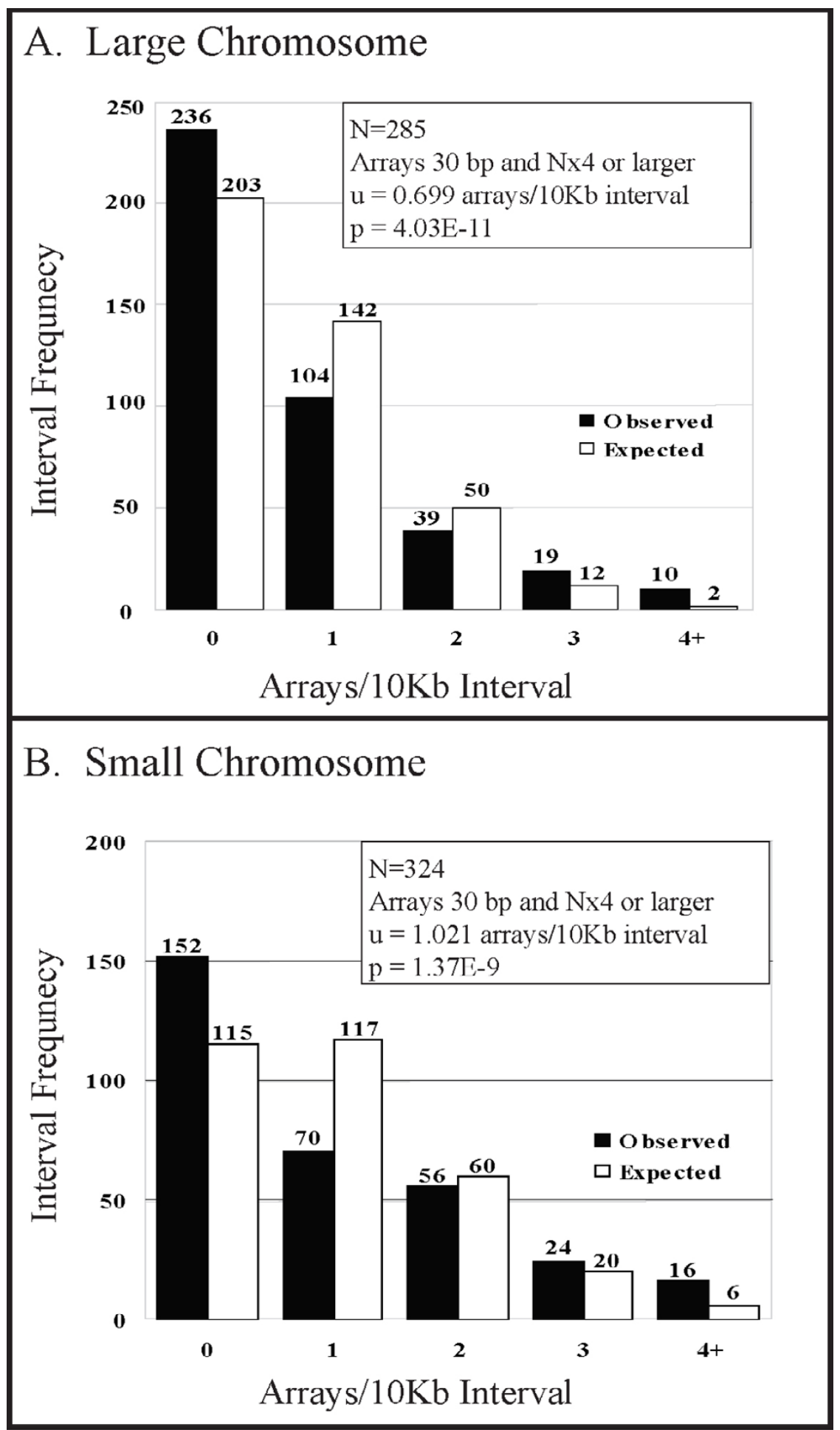

Figure 3

Repeat array distribution Goodness-of-fit test against a Poisson distribution. The bar graphs in each of the panels indicate the observed and expected number of $10 \mathrm{Kbp}$ intervals containing zero, one, two, three and four or more repeat arrays for the $B$. pseudomallei large (A) and small (B) chromosomes. For each chromosome, the total number of arrays, average arrays/interval used to generate the Poisson expected frequencies, and calculated $p$ values are shown. Values above each bar indicate the observed or expected frequencies in each category. 
Table 2: VNTR primer sequences and concentrations

\begin{tabular}{|c|c|c|c|c|}
\hline Locus Name & Primer Sequence & PCR Mix & Final [Primer] uM & Dye \\
\hline \multirow[t]{2}{*}{$933 \mathrm{k}$} & F: atggtggcggccgtcggcgaaaacc & I.I & $0.20 *$ & Fam \\
\hline & R: gctcgaatgggtgtacgaagggccacgctgattc & & 0.2 & \\
\hline \multirow[t]{2}{*}{$2065 \mathrm{k}$} & F: gggggacccggcgcacgacagg & 1.1 & $0.20 * *$ & Vic \\
\hline & R: cggcgcgttgggacgatcggcttgat & & 0.2 & \\
\hline \multirow[t]{2}{*}{297 I k } & F: gcgcaagcgcgactcggccactcg & 1.2 & 0.1 & Pet \\
\hline & R: gtcgccgggcgcggggctacatcttctta & & 0.1 & \\
\hline \multirow[t]{2}{*}{$3 \mid 45$ k } & F: ggcaggcaccgccggcatggaagc & 1.2 & 0.2 & $\mathrm{Ned}$ \\
\hline & R: gcgtcgcgcgtatcgatccgactgattgtacc & & 0.2 & \\
\hline \multirow[t]{2}{*}{$2666 k^{b}$} & F: gctgcaagtccgccttcacgcgcatcag & 2 & 0.13 & $\mathrm{Ned}$ \\
\hline & R: gcggcggccggctcgagttggact & & 0.13 & \\
\hline \multirow[t]{2}{*}{$367 / k^{a}$} & F: gcagcggctttggatcgcccgggttct & 2 & $0.10 *$ & Pet \\
\hline & R: gggccggggcgcggaagtcgaaagtt & & 0.1 & \\
\hline \multirow[t]{2}{*}{$21 / 5 k^{a}$} & F: ggtgcgtgctggtgtcgctgctgtgctatctgt & 2 & 0.1 & Vic \\
\hline & R: ggggaaggcgccggattgcccgagtt & & 0.1 & \\
\hline \multirow[t]{2}{*}{$2341 \mathrm{k}$} & F: ggcttcgcacccgccccatttcagc & 2 & $0.10 * *$ & Fam \\
\hline & R: gcaccgggcgcggcgcactcg & & 0.1 & \\
\hline \multirow[t]{2}{*}{$1500 \mathrm{k}$} & F: cagagcgcggcgaggacgatcaaaaggag & 2 & $0.10 * *$ & Fam \\
\hline & R: gccgcggctactggcgccaccattg & & 0.1 & \\
\hline \multirow[t]{2}{*}{309 I k } & F: aattcgtcggcagcgggcacggaagatg & 3 & $0.20 *$ & Vic \\
\hline & R: agcgggcacgcagcttgacggaacc & & 0.2 & \\
\hline \multirow[t]{2}{*}{$3152 k^{c}$} & F: cggcgcggcgttcgtccggctactc & 3 & 0.2 & Pet \\
\hline & R: acgaatgcggggcccgaggttgacgatagg & & 0.2 & \\
\hline \multirow[t]{2}{*}{$3652 \mathrm{k}$} & F: gattcggacggtcggccccgggtatcaa & 3 & 0.25 & $\mathrm{Ned}$ \\
\hline & R: gctggacgaaatccggggcgggacaaag & & 0.25 & \\
\hline \multirow[t]{2}{*}{$3564 \mathrm{k}$} & F: ggccatgccgctgccgggttgagc & 3 & $0.20 *$ & Fam \\
\hline & R: cgcgggaagcgggttttgacgaagggtgtagttt & & 0.2 & \\
\hline \multirow[t]{2}{*}{$20 \mathrm{k}$} & F: gcaccgcgagcgccgagcccgaac & 4 & $0.20 *$ & Ned \\
\hline & R: gcgcccggcggccaaccctttgtcg & & 0.2 & \\
\hline \multirow[t]{2}{*}{$857 k$} & F: cgcgccggatacgccgtccaccag & 4 & 0.2 & Fam \\
\hline & R: acgccggcgccgcaatggctgtc & & 0.2 & \\
\hline \multirow[t]{2}{*}{$1690 \mathrm{k}$} & F: cgtttcccgtttgatgcatttgcgttccctttgaa & 4 & 2 & Pet \\
\hline & R: catcgcggccgtcagaaaagttgagaaacctcgtc & & 2 & \\
\hline $2445 k$ & F: caggccgggccgtcgacgtgttcg & 4 & 0.1 & Vic \\
\hline
\end{tabular}


Table 2: VNTR primer sequences and concentrations (Continued)

\begin{tabular}{|c|c|c|c|c|}
\hline & R: atcggggagggagggcgacgaggtgaagg & & 0.1 & \\
\hline \multirow[t]{2}{*}{$1367 \mathbf{k}^{\mathrm{a}}$} & F: ggcgctgccgtggccggacgac & 5 & 0.3 & Ned \\
\hline & R: gccggcgaagcatcgaggcggtatg & & 0.3 & \\
\hline \multirow[t]{2}{*}{$1764 \mathrm{k}$} & F: acccggtcggcacgctacggaactggttgtt & 5 & 2 & Pet \\
\hline & R: cggcggtgaactggcttggcggacctc & & 2 & \\
\hline \multirow[t]{2}{*}{$2815 \mathrm{k}$} & F: cgaggacgcggctcaggtcgatgattttcagg & 5 & 0.1 & Fam \\
\hline & R: cggcgggcgggctttgcatgtcgt & & 0.1 & \\
\hline \multirow[t]{2}{*}{$2170 \mathrm{k}$} & F: cgcatcggcgcaacgtcgtcatctcgt & 6.1 & $0.10 *$ & Fam \\
\hline & R: cggcgaccgcgcagggcagttga & & 0.1 & \\
\hline \multirow[t]{2}{*}{$389 k$} & F: gttacaagcgcgggtcggcaagaggctgaaa & 6.1 & $0.10 *$ & Vic \\
\hline & R: gccggtgttgaacgagtgggtggcgtaagc & & 0.1 & \\
\hline \multirow[t]{2}{*}{$1788 \mathrm{k}$} & F: gcgcggcgagaacggcaagaacgaa & 6.2 & $0.10 *$ & Pet \\
\hline & R: gagcatcgggtggggcggcgcgtattgat & & 0.1 & \\
\hline \multirow[t]{2}{*}{$1217 \mathrm{k}^{\mathrm{a}}$} & F: gcgagatgcgggcgtgtgcggtgtg & 6.2 & $0.2^{* *}$ & Ned \\
\hline & R: gcggcggccgtgagcctgctgagaatc & & 0.2 & \\
\hline \multirow[t]{2}{*}{$397 \mathrm{k}$} & F: cgcacgcggggcaggccgagacg & 7 & $0.20 * *$ & Fam \\
\hline & R: gcggtcgcgeccttccacgcttcatc & & 0.2 & \\
\hline \multirow[t]{2}{*}{$2050 \mathrm{k}$} & F: ccggcggccgcttcgtcgtctcg & 7 & 0.2 & Pet \\
\hline & R: cgcgaagtcgatccgcaactgcctgctcac & & 0.2 & \\
\hline \multirow[t]{2}{*}{$2862 \mathbf{k}^{\mathrm{a}}$} & F: gattcggcgcggtccgtaccagcttgttgc & 7 & 0.3 & Vic \\
\hline & R: gcgcggggtatgtgacggggcagagc & & 0.3 & \\
\hline \multirow[t]{2}{*}{$140 k^{a}$} & F: gcgcgcaccggccgcttcgactgacga & 8 & 0.3 & Fam \\
\hline & R: gcatacggtcgcgccgggcgggtggtaggaag & & 0.3 & \\
\hline \multirow[t]{2}{*}{$2356 \mathrm{k}$} & F: ccgctgatcggcgtgctgacggtgtt & 8 & 0.2 & $\mathrm{Ned}$ \\
\hline & R: gctcggggcgctcggcgttctctg & & 0.2 & \\
\hline \multirow[t]{2}{*}{$2518 \mathbf{k}^{\mathbf{a}}$} & F: caggcgcagttgtcgattgacgggtgtggac & 8 & 0.2 & Vic \\
\hline & R: acggcgggatgtgcgcggtctgacg & & 0.2 & \\
\hline \multirow[t]{2}{*}{$2124 k^{a}$} & F: ctgcgcgtgctgcccggcgtcac & 9 & 0.2 & Vic \\
\hline & R: cgcgtggcggaatgcgcatgatagg & & 0.2 & \\
\hline \multirow[t]{2}{*}{$1934 k^{c}$} & F: cgacgtgatccgcggctatctcgaagacg & 9 & 0.2 & Pet \\
\hline & R: ccgacgcggcttgccagcttggatcgttag & & 0.2 & \\
\hline
\end{tabular}

* $50 \%$ unlabeled Forward primer

** $75 \%$ unlabeled Forward primer

a Not recommended for globally diverse isolates

b Not used in phylogenetic analysis due to $<80 \%$ amplification

c Locus reported in Liu et al. 2006 [22] 
Table 3: MLVA loci characteristics

\begin{tabular}{|c|c|c|c|c|c|c|c|c|}
\hline Chromosome & $\begin{array}{l}\text { VNTR Locus } \\
\text { Name }\end{array}$ & $\begin{array}{l}\text { Array start } \\
\text { position in } \\
\mathrm{K} 96243\end{array}$ & $\begin{array}{l}\text { Consensus } \\
\text { Repeat } \\
\text { sequence }\end{array}$ & In CDS & $\begin{array}{c}\text { Array Size in } \\
\text { K96243 } \\
\text { (bpxcopy\#) }\end{array}$ & $\begin{array}{l}\text { Amplicon Size } \\
\text { Range (bp) }\end{array}$ & $\begin{array}{c}\text { Number of } \\
\text { Alleles }\end{array}$ & Nei's Diversity \\
\hline Large & $933 \mathrm{k}$ & 933861 & $\underset{\mathrm{AA}}{\text { CGGCGAGGGA }}$ & no & $12 \times 10$ & $160-365$ & 16 & 0.89 \\
\hline Small & 2065 k & 2064726 & TCGAGTCA & no & $8 \times 8$ & $238-370$ & 21 & 0.9 \\
\hline Large & 297 I k & 2971247 & CGTGCTT & no & $7 \times 9$ & $20|-3| 4$ & 22 & 0.92 \\
\hline Large & $3 / 45 \mathrm{k}$ & 3144932 & ССТTCСТCG & no & $9 \times 8$ & $220-345$ & 14 & 0.86 \\
\hline Large & $2666 k$ & 2666129 & CTTTCGCTA & yes & $9 \times 7$ & $268-332$ & 8 & 0.79 \\
\hline Large & $3671 \mathrm{k}$ & 3671327 & CTTGGAC & no & $7 \times 21$ & $205-364$ & 23 & 0.93 \\
\hline Small & $2115 \mathrm{k}$ & 2115424 & CGCCGGTT & no & $8 \times 15 d$ & $290-399$ & 15 & 0.83 \\
\hline Large & 234 I k & 2340566 & TTCGTGCGC & no & $9 \times 7$ & $122-219$ & 10 & 0.8 \\
\hline Large & $1500 \mathrm{k}$ & 1500968 & GGGAAAGTGCG & no & $11 \times 6$ & $312-379$ & 7 & 0.55 \\
\hline Small & $3091 \mathrm{k}$ & 3091444 & TCACGGC & no & $7 \times 12$ & $202-287$ & II & 0.86 \\
\hline Large & 3152 k & 3152382 & GACTCG & no & $6 \times 17$ & $|60-37|$ & 26 & 0.94 \\
\hline Large & $3652 \mathrm{k}$ & 3651903 & CCGTAGTC & no & $8 \times 8$ & $320-408$ & 13 & 0.87 \\
\hline Large & $3564 k$ & 3563188 & $\begin{array}{c}\text { GCAGCCTTCTT } \\
\text { CGCG }\end{array}$ & yes & $15 \times 30 d$ & $295-692$ & 10 & 0.63 \\
\hline Large & $20 \mathrm{k}$ & 20292 & CGCCTCA & no & $7 \times 10$ & $245-435$ & 22 & 0.92 \\
\hline Small & 857 k & 857207 & CGAAYGAGC & no & $9 \times 11$ & $209-300$ & 12 & 0.81 \\
\hline Small & $1690 \mathrm{k}$ & 1689945 & CGTCGATA & no & $8 \times 13$ & $252-405$ & 13 & 0.78 \\
\hline Small & $2445 k$ & 2444540 & GGCACTTC & no & $8 \times 19$ & $205-391$ & 19 & 0.89 \\
\hline Small & $1367 \mathrm{k}$ & 1366924 & CGCRTCGAA & yes & $9 \times 24$ & $454-686$ & 26 & 0.92 \\
\hline Small & 1764 k & 1764166 & $\underset{T}{\text { GCCGCTGAAGT }}$ & no & $12 \times 20$ & $233-466$ & 12 & 0.47 \\
\hline Large & $2815 \mathrm{k}$ & 2815153 & TGGCGTCTT & yes & $9 \times 7$ & $223-439$ & 19 & 0.86 \\
\hline Large & $2170 \mathrm{k}$ & 2171435 & ATGCCGTGG & no & $9 \times 24$ & $229-513$ & 25 & 0.93 \\
\hline Small & $389 \mathrm{k}$ & 388768 & GACGAACC & no & $8 \times 6$ & $224-313$ & 12 & 0.87 \\
\hline Small & $1788 \mathrm{k}$ & 1788368 & $\begin{array}{c}\text { GTCGTGCGATC } \\
\text { CTGCT }\end{array}$ & no & $16 \times 8$ & $203-367$ & 11 & 0.86 \\
\hline Large & $1217 \mathrm{k}$ & 1217379 & CGGACCTAGG & no & $10 \times 15$ & $357-480$ & 14 & 0.85 \\
\hline Small & $397 \mathrm{k}$ & 397146 & GCCCGAGA & no & $8 \times 12$ & $226-401$ & 17 & 0.88 \\
\hline Small & $2050 \mathrm{k}$ & 2049749 & $\begin{array}{l}\text { CGATGCGGT/ } \\
\text { GCACCCAAC }\end{array}$ & yes/yes & $9 \times 8 / 9 \times 8$ & $377-549$ & 18 & 0.92 \\
\hline Small & $2862 k$ & 2861834 & CTCGCCTTTG & no & $10 \times 8$ & $273-422$ & 15 & 0.88 \\
\hline Large & $140 \mathrm{k}$ & 139952 & GCGCCGAA & no & $8 \times 15$ & $367-675$ & 28 & 0.93 \\
\hline Large & $2356 k$ & 2356018 & CTTGGCGA & no & $8 \times 13$ & $236-425$ & 16 & 0.9 \\
\hline Small & $2518 \mathrm{k}$ & 2517929 & CCGCGAT & no & $7 \times 31$ & $294-394$ & 17 & 0.92 \\
\hline Small & $2124 k$ & 2123866 & CCTTCGCG & no & $8 \times 23$ & $332-490$ & 14 & 0.88 \\
\hline Small & $1934 \mathrm{k}$ & 1933513 & $\begin{array}{c}\text { CGAGTCGGCG } \\
\text { GTT }\end{array}$ & no & $13 \times 16$ & $224-645$ & 21 & 0.91 \\
\hline
\end{tabular}

MLVA loci, there were more mutations observed for large chromosome loci than small chromosome loci. Additionally, there was a nonsignificant trend towards multiple repeat mutations ( $\mathrm{p}=0.5127)$, as well as, a nominally significant trend towards deletion mutations $(\mathrm{p}=0.0495)$ (Table 6). The multiple repeat mutations ranged from 2 to 6 repeat units. Two of the duplicated loci $(1558 \mathrm{k}$ and $3851 \mathrm{k}$ ), had less than 50\% PCR amplification. Highly unpredictable PCR amplification was seen with three loci (3166 k, $1343 \mathrm{k}$ and $2646 \mathrm{k}$ ). These PCR failures could be due to the difficult nature of PCR in a high GC organism such as $B$. psuedomallei, or could be indicative of loss of priming sites due to recombination. The PCR amplification success rates for the remaining loci were comparable to the MLVA loci. The duplicated loci averaged 2.6 mutations/locus, and combined mutation rate for 15 duplicated tandem repeat loci was also comparable to the nonduplicated MLVA loci, at $1.23 \times 10^{-3}$ for $\sim 18,000$ generations.

\section{Discussion}

Burkholderia pseudomallei is a distinctive microbial pathogen due to its ability to survive and exploit a wide variety of environmental conditions, as well as, the opportunistic infection of animals. It can cause mild, chronic, or rapidly progressing and potentially fatal disease states in a range of animal hosts [33], and it has a demonstrated ability invade the cells of other eukaryotic organisms such as fungi and amoeba $[34,35]$. It has been known to survive extreme environmental conditions for long periods of time, including nutrient starvation [36], and chlorine concentrations generally recognized as sufficient for potable water treatment [37]. This level of environmental flexibility and pathogenic potential may require the $B$. pseudomallei genome to be highly plastic in order to quickly adapt to different environments. Indeed, while the large chromosome primarily harbors genes essential for growth, the small chromosome contains more diverse genes that are primarily involved in survival and/or exploiting variable 
Table 4: B. pseudomallei and B. mallei isolates

\begin{tabular}{|c|c|c|c|c|c|c|c|}
\hline Species & Strain Name & Other Identifier & Country of Origin & Source & Site & Date & Tree Code \\
\hline \multirow[t]{45}{*}{ B. pseudomallei (66) } & PHLS 5* & 2002721617, NCTC 8016 & Australia & Sheep & & 1949 & Bp_Aust_Sheep_49 \\
\hline & PHLS 9I & $2002721622,84-1097$ & Australia & Sheep & Lung & 1984 & Bp_Aust_Sheep_84 \\
\hline & PHLS 92 & $2002721623,85-1097$ & Australia & Cow & Spleen & 1985 & Bp_AustCow_85 \\
\hline & PHLS 83 & & Australia & Environment & Soil & & Bp_Austl_Env \\
\hline & PHLS 84* & & Australia & Environment & Soil & & Bp_Aust2_Env \\
\hline & PHLS 85 & & Australia & Environment & Soil & & Bp_Aust3_Env \\
\hline & PHLS 104 & & Australia & Goat & Lymph node & & Bp_Aust_Goat \\
\hline & $146^{*}$ & & Australia & Animal & Right Udder & 1992 & Bp_Aust_NT_Animal_I_92 \\
\hline & 147 & & Australia & Animal & Med Lymph Node & 1992 & Bp_Aust_NT_Animal_2_92 \\
\hline & 213 & & Australia & Environmental & Soil & 1993 & Bp_Aust_NT_Envl_93 \\
\hline & 214 & & Australia & Environmental & Soil & 1993 & Bp_Aust_NT_Env2_93 \\
\hline & $465 a$ & & Australia & Human & Blood & 1997 & Bp_Aust_NT_Human_I_97 \\
\hline & $465 \mathrm{e}$ & & Australia & Human & Sputum & 1997 & Bp_Aust_NT_Human_2_97 \\
\hline & 1459 & & Australia & Human & Sputum & 2002 & Bp_Aust_NT_Human_02 \\
\hline & 1627 & & Australia & Human & Sputum & 2003 & Bp_Aust_NT_Human_I_03 \\
\hline & 1628 & & Australia & Human & Throat & 2003 & Bp_Aust_NT_Human_2_03 \\
\hline & PHLS 6 & & Bangledesh & Human & & 1960 & Bp_Bangledesh_Human_60 \\
\hline & PHLS 208 & & Ecuador & Human & & & Bp_Equador_Human \\
\hline & PHLS 68 & & Fiji & Human & Blood & 1992 & Bp_Fiji_Human_92 \\
\hline & PHLS 33 & 2002721630,7605 & France & Environment & Manure & 1976 & Bp_France_Env_76 \\
\hline & PHLS 24 & $200272|620,764|$ & France & Horse & Stool & 1976 & Bp_France_Horse_76 \\
\hline & PHLS 4075 & & Holland (tourist) & Human & Sputum & 1999 & Bp_tourist_2_99 \\
\hline & PHLS 4I52 & & Holland (tourist) & Human & Cervix & 1999 & Bp_tourist_3_99 \\
\hline & PHLS I7 & & Indonesia & Monkey & Spleen & 1990 & Bp_Indol_Monkey_90 \\
\hline & PHLS I8* & & Indonesia & Monkey & Pus & 1990 & Bp_Indo2_Monkey_90 \\
\hline & PHLS 3477 & & $\begin{array}{c}\text { Italy } \\
\text { (Tourist SE Asia) }\end{array}$ & Human & Sputum & 1998 & Bp_Touristl_98 \\
\hline & PHLS $3 I^{*}$ & & Kenya & Environment & Water drain & 1992 & Bp_Kenya_Env_92 \\
\hline & PHLS 25* & & Madagascar & Environment & Soil & 1977 & Bp_Madagascar_Env_77 \\
\hline & PHLS 7I & & Malaysia & Human & & & Bp_Malaysia I_Human \\
\hline & PHLS 72* & & Malaysia & Human & & & Bp_Malaysia2_Human \\
\hline & PHLS 73 & & Malaysia & Human & & & Bp_Malaysia3_Human \\
\hline & PHLS 79 & & Malaysia & Human & & & Bp_Malaysia4_Human \\
\hline & PHLS 75* & & Malaysia & Human & & & Bp_Malaysia5_Human \\
\hline & PHLS 9 & 2002721637,521 & Pakistan & Human & & 1988 & Bp_Pakistan_Human_88 \\
\hline & PHLS 16 & & Phillipines & Monkey & & 1990 & Bp_PhillipinesI_Monkey_90 \\
\hline & PHLS I4 & & Phillipines & Monkey & Liver & 1990 & Bp_Phillipines2_Monkey_90 \\
\hline & PHLS 39* & & Singapore & Human & Blood & 1988 & Bp_SingI_Human_88 \\
\hline & PHLS 36 & 2002721635 & Singapore & Human & & 1988 & Bp_Sing2_Human_88 \\
\hline & PHLS 38 & & Singapore & Human & & 1988 & Bp_Sing3_Human_88 \\
\hline & PHLS 40 & & Singapore & Human & & 1988 & Bp_Sing4_Human_88 \\
\hline & PHLS 19 & & Singapore & Environment & & 1991 & Bp_Sing_Env_91 \\
\hline & PHLS 3584 & & $\begin{array}{l}\text { Sweden } \\
\text { (Tourist SE Asia) }\end{array}$ & Human & Blood & 1998 & Bp_Tourist2_98 \\
\hline & PHLS 8* & & Thailand & Human & & 1988 & Bp_Thai_Human_88 \\
\hline & PHLS 20 & & Thailand & Human & Blood & 1990 & Bp_Thai_Human_90 \\
\hline & PHLS 53 & $2002721633,307 a$ & Thailand & Human & Urine & 1987 & Bp_Thail_NE_Human_87 \\
\hline
\end{tabular}


Table 4: B. pseudomallei and B. mallei isolates (Continued)

\begin{tabular}{|c|c|c|c|c|c|c|c|}
\hline & PHLS 43 & & Thailand & Human & & 1988 & Bp_Thail_NE_Human_88 \\
\hline & PHLS 45 & & Thailand & Human & & 1988 & Bp_Thai2_NE_Human_88 \\
\hline & PHLS 47 & & Thailand & Human & & 1988 & BP_Thai4_NE_Human_88 \\
\hline & PHLS 44* & & Thailand & Human & & 1988 & Bp_Thai5_NE_Human_88 \\
\hline & PHLS 392 & & Thailand & Human & & 1989 & Bp_Thai_NE_Human_89 \\
\hline & PHLS 216 & 2002721626 & Thailand & Environment & & 1990 & Bp_Thai_NE_Env_90 \\
\hline & PHLS IIO & & Thailand & Human & Urine & 1992 & Bp_Thail_NE_Human_92 \\
\hline & PHLS III & & Thailand & Human & Blood & 1992 & Bp_Thai2_NE_Human_92 \\
\hline & PHLS II $2 *$ & & Thailand & Human & & 1992 & Bp_Thai3_NE_Human_92 \\
\hline & $\begin{array}{l}\text { PHLS 98/SID } \\
\text { 2953* }\end{array}$ & & United Kingdom & Human & & 1998 & Bp_UK_Human I_98 \\
\hline & $\begin{array}{l}\text { PHLS 98/SID } \\
3292^{*}\end{array}$ & & United Kingdom & Human & & 1998 & Bp_UK_Human2_98 \\
\hline & 99/SID 4349 & & United Kingdom & Human & & 1999 & Bp_UK_Human_99 \\
\hline & PHLS 2889 & & $\begin{array}{l}\text { United Kingdom } \\
\text { (Bangledesh national) }\end{array}$ & Human & Sputum & 1998 & $\begin{array}{c}\text { Bp_Bangledesh_National_h } \\
\text { uman_98 }\end{array}$ \\
\hline & PHLS 38II & & $\begin{array}{c}\text { United Kingdom } \\
\text { (Bangledesh national) }\end{array}$ & Human & Abscess & 1999 & $\begin{array}{c}\text { Bp_Bangledesh_National_I } \\
\text { human_99 }\end{array}$ \\
\hline & PHLS 387I & & $\begin{array}{l}\text { United Kingdom } \\
\text { (Bangledesh national) }\end{array}$ & Human & Abscess & 1999 & $\begin{array}{c}\text { Bp_Bangledesh_National_2 } \\
\text { human_99 }\end{array}$ \\
\hline & PHLS 3783* & & $\begin{array}{l}\text { United Kingdom } \\
\text { (Tourist SE Asia) }\end{array}$ & Human & Sputum & 1999 & Bp_Touristl_99 \\
\hline & PHLS 35 & 2002721638, Ducrete & Vietnam & Human & & 1963 & Bp_Vietnam_Human_63 \\
\hline & PHLS I26 & & & & & & Bpl \\
\hline & ACTC 11668 & & & & & & Bp2 \\
\hline & ACTC 15682 & & & & & & Bm_Hungaryl_6I \\
\hline & ACTC 23343 & & & Type strain & & & Bp_TypeStrain \\
\hline \multirow[t]{21}{*}{ B. mallei (2I) } & ACTC 10399* & $\begin{array}{l}200272 \text { I } 275, \text { GBII } \\
\text { NCTC I }\end{array}$ & China & Horse & Lung & 1956 & Bm_China_Horse_56 \\
\hline & ACTC 15310 & & Hungary & & & 1961 & Bp3 \\
\hline & NCTC 10229 & GB5 & Hungary & & & 1961 & Bm_Hungary2_6I \\
\hline & NCTC 3708 & GB9 & India & Mule & Lung & 1932 & Bm_India_Mule_32 \\
\hline & NCTC 3709 & GBIO & India & Horse & & 1932 & Bm_India_Horse_32 \\
\hline & NCTC 10260 & GB6 & Turkey & Human & & 1949 & Bm_Turkey_Human_49 \\
\hline & NCTC 10248 & GB4 & Turkey & Human & & 1950 & Bm_Turkey_Human_50 \\
\hline & NCTC 10247 & GB7 & Turkey & & & 1960 & Bm_Turkey_60 \\
\hline & NCTC 120 & GB3 & United Kingdom & & & 1920 & Bm_UK_1920 \\
\hline & 85_503 & & & Equine & & & Bm_equine \\
\hline & 86_567 & & East India & Mule & & & $\mathrm{Bml}$ \\
\hline & ISU & & & & & & $\mathrm{Bm} 2$ \\
\hline & Turkey_I & & Turkey & & & & Bm_Turkeyl \\
\hline & Turkey_2 & & Turkey & & & & Bm_Turkey2 \\
\hline & Turkey_3 & & Turkey & & & & Bm_Turkey3 \\
\hline & Turkey_4 & & Turkey & & & & Bm_Turkey4 \\
\hline & Turkey_5 & & Turkey & & & & Bm_Turkey5 \\
\hline & Turkey_6 & & Turkey & & & & Bm_Turkey6 \\
\hline & Turkey_7 & & Turkey & & & & Bm_Turkey7 \\
\hline & Turkey_8 & & Turkey & & & & Bm_Turkey8 \\
\hline & Turkey_9 & & Turkey & & & & Bm_Turkey9 \\
\hline
\end{tabular}

* Isolates used in the screening panel. 


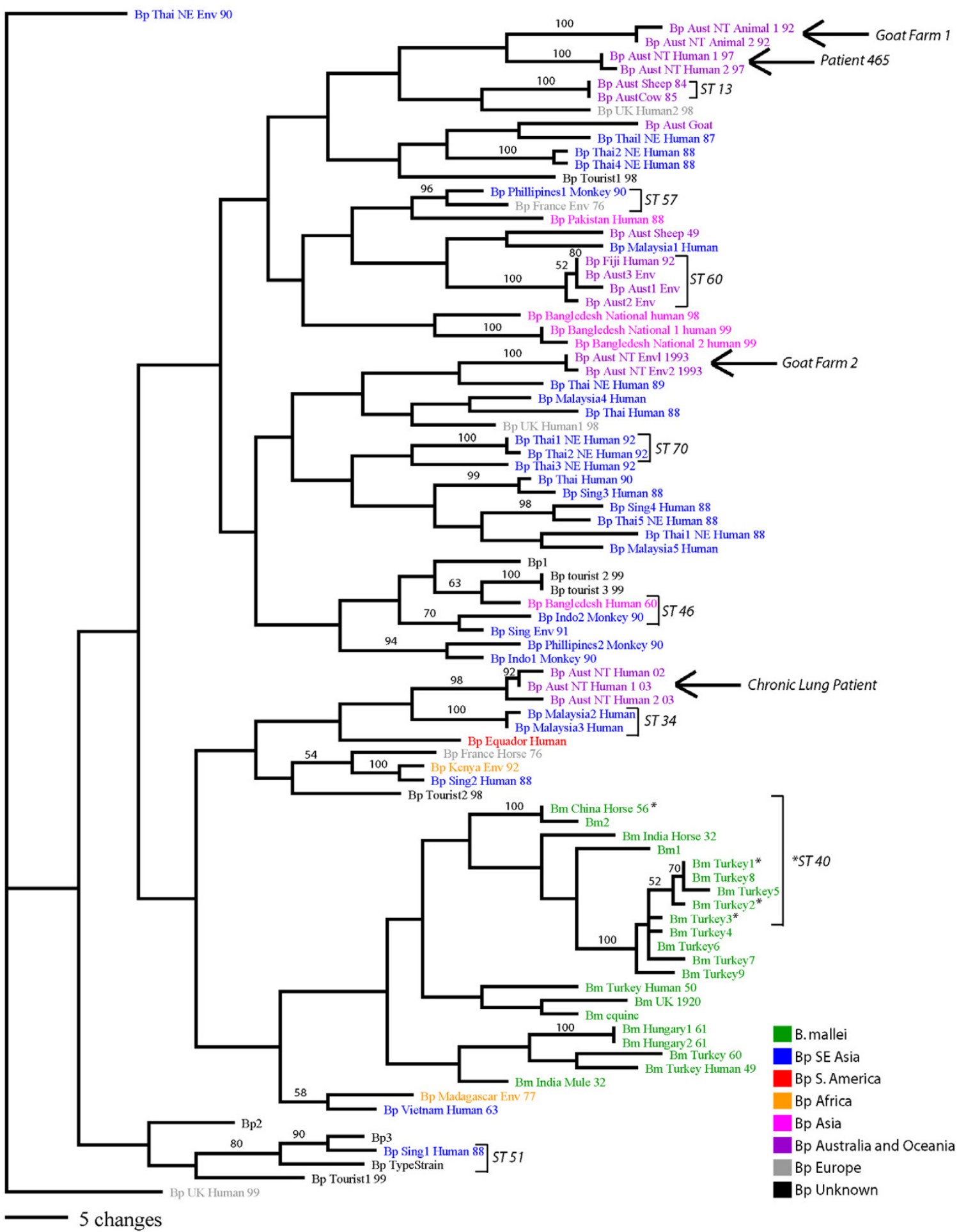

Figure 4

Arbitrarily rooted phylogram of 66 B. pseudomallei and 2 I B. mallei isolates. Colors indicate the geographic area from which the isolates were collected. Arrows indicate isolates from patients or from a specific outbreak event. Isolates that had identical MLST genotypes are bracketed and the sequence type is given. * indicates which B. mallei isolates were available on the MLST database. 
Table 5: B. pseudomallei' MLVA loci mutation rate

\begin{tabular}{|c|c|c|c|c|c|c|c|c|c|c|}
\hline $\begin{array}{l}\text { Locus } \\
\text { Name }\end{array}$ & Chromosome & $\begin{array}{c}\text { Inside } \\
\text { CDS }\end{array}$ & Array size* & $\begin{array}{c}\text { Total } \\
\text { Number of } \\
\text { Mutations }\end{array}$ & Insertions & Deletions & $\begin{array}{l}\text { Single Repeat } \\
\text { Changes }\end{array}$ & $\begin{array}{c}\text { Multiple Repeat } \\
\text { Changes }\end{array}$ & Lineages** & $\begin{array}{c}\text { Mutation } \\
\text { Rate }\end{array}$ \\
\hline $1788 \mathrm{k}$ & Small & no & $16 \times 9$ & 1 & - & 1 & 1 & - & 94 & $5.3 \times 10^{-5}$ \\
\hline $2862 k$ & Small & no & $10 \times 11$ & 3 & 3 & - & 2 & 1 & 90 & $1.7 \times 10^{-4}$ \\
\hline $1367 \mathrm{k}$ & Small & yes & $9 \times 22$ & 1 & - & 1 & 1 & - & 75 & $6.7 \times 10^{-5}$ \\
\hline $3 / 45 k$ & Large & no & $9 \times 13$ & 1 & 1 & - & 1 & - & 84 & $6.0 \times 10^{-5}$ \\
\hline $2170 k$ & Large & no & $9 \times 12$ & 4 & 4 & - & 4 & - & 89 & $2.3 \times 10^{-4}$ \\
\hline $1690 \mathrm{k}$ & Small & no & $8 \times 13$ & 2 & - & 2 & 2 & - & 93 & $1.1 \times 10^{-4}$ \\
\hline $933 \mathrm{k}$ & Large & no & $12 \times 14$ & 1 & - & 1 & 1 & - & 91 & $5.5 \times 10^{-5}$ \\
\hline $2065 k$ & Small & no & $8 \times 14$ & 1 & 1 & - & 1 & - & 95 & $5.3 \times 10^{-5}$ \\
\hline $2050 \mathrm{k}$ & Small & yes & $9 \times 16$ & 1 & - & 1 & 1 & - & 93 & $5.4 \times 10^{-5}$ \\
\hline $2518 \mathrm{k}$ & Small & no & $7 \times 22$ & 2 & 2 & - & 2 & - & 90 & $1.1 \times 10^{-4}$ \\
\hline $3152 \mathrm{k}$ & Large & no & $6 \times 25$ & 2 & 2 & - & 2 & - & 95 & $1.1 \times 10^{-4}$ \\
\hline $2815 k$ & Large & yes & $9 \times 19$ & 1 & 1 & - & 1 & - & 94 & $5.3 \times 10^{-5}$ \\
\hline Total & & & & 20 & 14 & 6 & 19 & I & † 92.50 & $1.113 \times 10^{-3}$ \\
\hline
\end{tabular}

'An isolate from the Arizona department of health (Bp9905-1902) was used for this study.

**Number of lineages successfully amplified out of 95 total

$\uparrow$ Average number of lineages to amplify

or contingent environmental conditions. Consequently, it is not biologically surprising that numerous genetic typing methodologies $[6,14,15,22]$, including the MLVA system reported here, find very high levels of genetic diversity within this organism. The high level of genetic diversity and host flexibility of the organism suggest enhanced mechanisms for generating and maintaining adaptive variation through processes such as selection, recombination and mutation.

The unusually high number of tandem repeats in $B$. pseudomallei (compared to other pathogenic bacteria with similarly sized genomes such as $B$. anthracis and Y. pestis, and other bacteria of similar GC content [5]) is indicative of potentially high genomic diversity which, in turn, may facilitate rapid genomic adaptation to a variable environment. While the majority of large VNTRs in B. pseudomallei are located intergenically and thus may have no direct phenotypic effect, it has been observed in other bacteria that such loci, when upstream of genes, can alter important biological functions through mechanisms such as transcriptional regulation and amino acid changes [3841]. Within coding regions we observed fewer tandem repeat arrays. The majority of these tandem arrays contain repeat units in multiples of three, which indicates the potential for adaptive variation. For example, Nierman et al. [5] observed variation in triplet repeat unit simple sequence repeat (SSR) loci that are located inside four genes coding for surface or putative virulence proteins in $B$. mallei and B. pseudomallei. A subsequent serial passage experiment of $B$. mallei through several mammalian hosts revealed indels in seven intragenic SSR loci, five of which caused frameshift mutations, while the other two were triplet repeats that only added or removed amino acids from the encoded protein [42]. This variation is consistent with the potential for phase variation during the infection cycle and may be a mechanism to avoid host defenses $[5,42]$. Thus, given the similarity of B. mallei and B. pseudomallei, the unusually high number of tandem repeat loci in $B$. pseudomallei, as well as their non-random arrangement, as indicated by a deviation from the expected Poisson distribution (Figure 3), may indicate that coding and non-coding genomic regions use different molecular mechanisms to adapt to different selective pressures.

In addition to the large number of tandem repeats in $B$. pseudomallei, there was a prevalence of duplicated tandem repeats throughout the genome. In B. pseudomallei, 37.9\% of tandem repeats in the large chromosome and $35.2 \%$ of tandem repeats in the small chromosome are found to be duplicated, at least in part, at other intra- and inter-chromosomal locations. Moreover, a serial passage experiment revealed that the duplicated loci show a contrasting trend towards deletions, as well as an increased frequency of multiple repeat changes in comparably sized repeat arrays, while displaying comparable mutation rates to non-duplicated loci; which is in contrast to the lack of bias in $Y$. pestis [43]. This suggests that the repeat regions within $B$. pseudomallei may facilitate large scale genomic rearrangements through recombination rather than slipstrand mispairing [44]. Although this has not been specifically studied in B. pseudomallei, it has been suggested that SSRs in Mycoplasma genomes may in fact facilitate genomic rearrangements via recombination [45], and that long tracts of tandem repeats may facilitate gene transfer [46]. Conversely, tandem repeats may not directly cause recombination, but rather be associated with regions that are prone to recombination for other reasons. Since recombination frequency is affected by the length of the homology between two loci [47] which in turn is control- 
Table 6: B. pseudomallei' duplicated loci mutation rates

\begin{tabular}{|c|c|c|c|c|c|c|c|c|c|c|c|c|}
\hline $\begin{array}{l}\text { Locus } \\
\text { Set }\end{array}$ & $\begin{array}{l}\text { Locus } \\
\text { Name }\end{array}$ & $\begin{array}{l}\text { Array start } \\
\text { position in } \\
\mathrm{K} 96243\end{array}$ & $\begin{array}{c}\text { Chromo- } \\
\text { some }\end{array}$ & $\begin{array}{c}\text { Inside } \\
\text { CDS }\end{array}$ & $\begin{array}{l}\text { Array } \\
\text { Size * }\end{array}$ & $\begin{array}{c}\text { Total } \\
\text { Number of } \\
\text { Mutations }\end{array}$ & Insertions & Deletions & $\begin{array}{c}\text { Single } \\
\text { Repeat } \\
\text { Changes }\end{array}$ & $\begin{array}{l}\text { Multiple } \\
\text { Repeat } \\
\text { Changes }\end{array}$ & Lineages $* *$ & $\begin{array}{c}\text { Mutation } \\
\text { Rate }\end{array}$ \\
\hline I & 1839 k & 1839378 & Large & no & $7 \times 2$ & 1 & - & 1 & 1 & - & 82 & $\frac{6.12 \times 10^{-}}{}$ \\
\hline I & $3166 \mathrm{k}$ & 3166431 & Small & no & - & - & - & - & - & - & - & \\
\hline 2 & I853 k & 1853384 & Large & no & $7 \times 27$ & 3 & - & 3 & 2 & 1 & 94 & $1.71 \times 10^{-}$ \\
\hline 2 & $2523 \mathrm{k}$ & 2523234 & Small & no & $7 \times 5$ & - & - & - & - & - & 88 & \\
\hline 2 & $817 k$ & 817412 & Small & no & $7 \times 17$ & 3 & - & 3 & - & 3 & 89 & $1.69 \times 10$ \\
\hline 3 & 1546 k & 1546409 & Large & no & $6 \times 20$ & - & - & - & - & - & 83 & \\
\hline 3 & $2620 k$ & 2620013 & Large & no & $6 \times 20$ & 3 & 1 & 2 & 3 & - & 93 & $1.64 \times 10^{-}$ \\
\hline 3 & 345 I k & 3451829 & Large & no & $6 \times 27$ & 1 & 1 & - & 1 & - & 94 & $\begin{array}{c}5.34 \times 10 \\
5\end{array}$ \\
\hline 3 & $3103 k$ & 3103500 & Small & no & $6 \times 27$ & 2 & 1 & 1 & 2 & - & 94 & $1.07 \times 10$ \\
\hline 4 & $200 \mathrm{k}$ & |9972| & Small & no & $7 \times 12$ & - & - & - & - & - & 94 & \\
\hline 4 & $735 \mathrm{k}$ & 734579 & Small & no & $6 \times 9$ & - & - & - & - & - & 92 & \\
\hline 5 & $1880 \mathrm{k}$ & 1879903 & Small & no & $7 \times 6$ & - & & - & - & - & 92 & \\
\hline 5 & $3984 k$ & 3983644 & Large & no & $7 \times 43$ & 7 & 3 & 4 & 3 & 4 & 94 & $\underset{4}{3.82} \times 10^{-}$ \\
\hline 6 & $1558 \mathrm{k}$ & I558336 & Large & no & $6 \times 11$ & - & - & - & - & - & 37 & \\
\hline 6 & $1343 \mathrm{~K}$ & 1343285 & Small & yes & - & - & - & - & - & - & - & \\
\hline 7 & 385 I k & 3851246 & Large & no & $7 \times 17$ & I & - & 1 & - & 1 & 41 & $1.25 \times 10^{-}$ \\
\hline 7 & $2646 \mathrm{~K}$ & 2646281 & Small & no & - & - & - & - & - & - & - & \\
\hline Total & & & & & & 21 & 6 & 15 & 12 & 9 & $\dagger 83.36$ & $\begin{array}{l}1.23 \times \\
10^{-3}\end{array}$ \\
\hline
\end{tabular}

'An isolate from the Arizona department of health (Bp9905-1902) was used for this study.

*Estimated array size in the $B$. pseudomallei strain used in the mutation rate study

**Number of lineages successfully amplified out of 95 total

† Average number of lineages to amplify

led by slip strand repair, the observed tandem repeat patterns could represent an interesting interaction between slip strand expansion and recombination.

During in vitro passage, mutation events were observed in multiple B. pseudomallei VNTR loci suggesting similar mutation rates at many loci. The MLVA combined mutation rate reported in this study is $1.113 \times 10^{-3}$ mutations/ generation, compared to combined MLVA rates in E. coli and $Y$. pestis rates of $6.4 \times 10^{-4}$ and $1.1 \times 10^{-3}$ mutations/ generation (respectively) $[26,43,48]$. The combined rate is, hence, comparable to those previously observed in $E$. coli and $Y$. pestis and offers similar subtyping discriminatory power. These rate calculations are dependent upon accurate estimation of the population growth parameters during serial passage and this may be particularly problematic for $B$. pseudomallei, which forms highly mucoid colonies. Experimental serial passage studies in E. coli and $Y$. pestis have previously identified a positive correlation between the in vitro mutation rate and natural locus diversity. This correlation was not detected in B. pseudomallei (analysis not shown) and it is not immediately obvious what differs between these pathogens. Perhaps due the much larger number of VNTR loci in B. pseudomallei, the current study was based upon an overwhelming number of equally and highly mutable loci, which are not commonly present in other genomes. In other words, the marker loci in E. coli and Y. pestis MLVA systems are stratified by their mutability but in the Burkholderia MLVA we may examining a number loci that are equally mutable. Thus, there is no correlation with array size. Another interesting difference is in the mutation products, where the majority (19:1) were single repeat changes. This bias was greater than observed in the E. coli and $Y$. pestis studies where the single-repeat mutational products were about $80 \%$ of the total observed. The lack of more two and three repeat changes needs to be explored in a larger in vitro population to see if this trend repeats reality in this particular genome.

Here we present a rapid PCR-based MLVA typing system using 32 independent VNTR loci. Although the initial development of a MLVA system in this organism was complicated by the quantity and duplicated nature of repeated regions found in $B$. pseudomalle $i$ and inconsistencies of the allelic size variation in comparison to the repeat unit size, we found 23 markers that were useful for phylogenetic analysis due to high diversity levels, minimal partial repeat differences and amplification success. An additional nine loci, while demonstrating some partial repeat 
sizes, are very useful for even finer scale resolution of closely related B. pseudomallei and B. mallei isolates from outbreak situations [49]. While no specific effort was made to design the MLVA primers specific to B. mallei, all B. mallei isolates tested amplified well at every locus, as expected given the phylogenetic relationship of the two species [6]. Conversely, B. thailandensis and B. cepacia did not amplify well in any of the loci, indicating that the MLVA loci primers will not support amplification in more distantly related bacterial species, although this has not been explicitly tested. Thus, this MLVA system represents a reliable method of identifying $B$. pseudomallei as well as B. mallei strains. Furthermore, this typing method is an easily transferable approach to high-resolution molecular typing analysis using low levels of crudely isolated DNA. The unique size and fluorescent label of each allele, as well as automated sizing software, allows for easy classification of each VNTR allele, and capillary electrophoresis significantly reduces run time.

Due to the relative effects of convergent evolution, reversal mutations, recombination, gene duplications and suggested horizontal gene transfer within Burkholderia pseudomallei, phylogenetic hypotheses have been difficult to establish. For example, neither MLST [6] nor MLVA are able to resolve the deeper relationships among distantly related $B$. pseudomallei isolates, as illustrated by the poor bootstrap support for deeper branches (Figure 4) and similar levels of consistency for a subset of the same isolates ( 0.63) (data not shown). This lack of resolution results in the absence of a geographic correlation within basal clades, although more derived clades do demonstrate geographic associations between isolates (Figure 4). In comparison, an analysis of Thai and Australian isolates using MLST exhibited no overlap between sequence types for the two countries [50]. However, phylogenetic analysis of these data lacks strong bootstrap values to support this geographic differentiation. Also, the analysis of historical isolates of $B$. pseudomallei using MLST reveals an overlapping sequence type between Australia and Thailand environmental isolates, and does not support the genetic distinction of isolates from Australia [51]. Thus, phylogenetic hypotheses using both MLVA and MLST data are difficult to establish with isolates that are geographically and temporally distant.

The present typing system targets VNTR loci over a wide range of diversity levels and consequently provides resolution between B. pseudomallei and B. mallei, while still providing high levels of discrimination between closely related isolates due to the high variability of tandem repeat loci in these bacterial pathogens. Whereas a number of typing methodologies such as PFGE, ribotyping, RAPDs and MLST have detected differences between isolates, their resolving power among very closely related isolates is less than MLVA $[6,14,15,19]$. For example, while MLST analysis provided only a single unique genotype for the B. mallei cluster, MLVA further resolved the B. mallei group into individual genotypes, even among very closely related isolates from Turkey with the same ribotype [32]. Additionally, B. pseudomallei isolates with the same sequence type often had different MLVA genotypes (Figure 4). This type of high resolution genotyping can define patterns of mutation within very closely related isolates from an outbreak, which can then be used for generating phylogenetic hypotheses [49].

A recent study by Liu et al. (2006) used six VNTR loci to differentiate $B$. pseudomallei isolates from an outbreak in Singapore [22]. Four of the six loci used were characterized in the present MLVA study. Two of these loci are included in this MLVA (Table 2), but the other two loci were found to be duplicated within the genome, and consequently were not included in MLVA development. This six-locus MLVA offered insight into the epidemiology of B. pseudomallei in Singapore, but presented limitations due to the lack of resolution inherent in agarose gel electrophoresis. Given the partial repeat sizes (as small as 3 bp) seen with capillary electrophoresis, it is doubtful that all alleles for these loci were detectable using agarose gels, and thus levels of diversity were underestimated. Additionally, because two of the VNTR loci that were used are duplicated within the genome, they are not recommended for phylogenetic analysis due to the confounding phylogenetic effects of gene duplication and associated possibilities for independent evolutionary trajectories.

\section{Conclusion}

In summary, the findings of this study suggest that the prevalence and location of tandemly repeated regions within the $B$. pseudomallei genome may generate and maintain adaptive variation in this bacterial pathogen. The intragenically located repeat regions, found twice as frequently on the "contingency-oriented" small chromosome [4], may provide for rapid changes in gene function. Duplicated repeat regions may facilitate genomic rearrangements which can lead to altered gene regulation. While the mutation rates of individual repeat regions do not appear to be enhanced over those in other organisms, the sheer number of these regions, some of which are quite large, provides great potential for genetic variation within this species.

Epidemiological characterization is important in any pathogen, but most especially for those that are emerging as global pathogens that may be exploited for biological terrorism, such as $B$. pseudomallei. While no typing system for $B$. pseudomallei can currently be used to reliably establish deep phylogeneic relationships, the $B$. pseudomallei-B. mallei multiplex MLVA typing system presented here pro- 
vides unsurpassed ability to resolve very closely related isolates, even those from the same patient. Efficient and sensitive genetic typing tools, such as the MLVA system presented here, are important for facilitating the increasingly important epidemiological and phylogenetic characterization of emerging pathogens.

\section{Methods \\ DNA preparation}

DNA for 66 B. pseudomallei and 21 B. mallei isolates was obtained from different institutions which used different extraction methods such as Dneasy (Qiagen, Valencia, CA) [52] and phenol/chloroform extraction [32] and quantified using a Pico Green quantification kit (Molecular Probes, Eugene, OR) and a minifluorometer (Turner Biosystems, Sunnyvale, CA). DNA was then normalized to $100 \mathrm{pg} / \mu \mathrm{L}$ for VNTR screening. Isolates for the global panel were selected to represent a wide variety of isolates in terms of geographic distribution, host source and date of isolation (Table 4).

\section{VNTR identification}

The complete genome sequence of Burkholderia pseudomallei strain K96243 was obtained from the National Center for Biotechnology Information [GenBank: NC 006350, NC 006351] and screened for potentially polymorphic repetitive sequences that were comprised of $\geq$ dinucleotide repeats, 4 copies and a total array size of $30 \mathrm{bp}$ using GeneQuest (Lasergene, Inc., Madison, WI) and Tandem Repeats Finder [53]. Primers flanking repeat sequences were designed using Primer Express (Lasergene, Inc., Madison, Wis.).

To assess the variability of repeated regions among a globally distributed set of isolates and to develop a comprehensive typing system for this organism, 104 repeated regions (48 from the large chromosome, 56 from the small) were targeted for analysis and subsequent incorporation into a multiple-locus VNTR analysis (MLVA) system. These VNTR loci were selected based upon PCR amplicon size, array size, locus duplication, and proximity to other arrays. Loci resulting in small PCR fragment sizes $(<1000 \mathrm{bp})$ were favored since such loci amplified better than larger regions, and are best suited for analytical platforms. Arrays with fewer than five copies of a motif were not selected for screening. Loci that were duplicated, either within or between chromosomes were also eliminated since multiple alleles would confuse a typing system. Lastly, repeat regions in close proximity (<1000 bp) to other repeat regions were avoided to preserve locus independence. Loci were not excluded based on their intra or intergenic location. The 104 candidate loci were examined for robust amplification and polymorphism across a screening panel which was comprised of $29 \mathrm{~B}$. pseudomallei isolates, one B. mallei isolate (ATCC 10399), and one
B. thailandensis isolate (ATCC 700388). B. pseudomallei stains in the screening panel included 15 closely related isolates from two different outbreaks in northern Australia [49], and 14 geographically diverse isolates from seven different countries (Table 4). This tiered screening panel allowed us to identify loci with varying degrees of polymorphism.

\section{VNTR screening using universal tail PCR and genotype analysis}

A high throughput five dye Universal Tail amplification and labeling methodology, developed for use in the low GC $(\mathrm{x}=35 \%)$ bacterium B. anthracis [54], was used to screen the chosen repeat region loci for variation among a combination of 29 diverse and closely related $B$. pseudomallei isolates. The optimal Tm for labeling sequences in B. anthracis is $55^{\circ} \mathrm{C}$, however due to the high G-C $(\mathrm{x}=$ $68.12 \%$ ) content of the B. pseudomallei genome, all PCR reactions were performed at a $\mathrm{Tm}$ of $72^{\circ} \mathrm{C}$.

The UT PCR labeling protocol provides for fluorescent labeling of any PCR amplicon with only four universal fluorescently labeled oligonucleotiodes. The fluorescently labeled universal primer is complimentary to a universal tailed primer sequence on the $5^{\prime}$ end of the target specific forward primer $(\mathrm{FAM}=$ ACCCAACTGAATAGAGAGC, NED = ATCGACTGTGTTAGGTCAC, PET = CTGTCCT TACCTCAATCTC and VIC = ACGCACTTGACTTGTCTTC) . This method significantly reduces the cost of initial screening by not having to order labeled primers for each locus.

PCR amplifications were performed using MJ Research 96-well DNA engines equipped with hot bonnets (BioRad, Waltham, MA). Reaction volumes equaled $10 \mu \mathrm{L}$ and contained the following: $10 \times$ Hot Master Taq buffer with $\mathrm{Mg}^{2+}$ (Brinkmann-Eppendorf, Westbury, New York), 200 $\mu \mathrm{M}$ deoxynucleoside triphosphates (Invitrogen, Carlsbad, CA), $5 \mu \mathrm{M}$ tailed primer, $50 \mu \mathrm{M}$ untailed primer, $50 \mu \mathrm{M}$ fluorescently labeled universal primer (Applied Biosystems, Foster City, CA), 1 U Hot Master Taq DNA Polymerase (Brinkmann-Eppendorf, Westbury, New York) and double-distilled $\mathrm{H}_{2} \mathrm{O}$. After an initial denaturation step at $94^{\circ} \mathrm{C}$ for $2 \mathrm{~min}, 30$ cycles of touchdown PCR were performed (denaturation at $94^{\circ} \mathrm{C}$ for $30 \mathrm{sec}$, annealing for 30 sec with an $0.5^{\circ} \mathrm{C} /$ cycle decrement at $72^{\circ} \mathrm{C}$, and an extension at $72^{\circ} \mathrm{C}$ for $30 \mathrm{sec}$ ) followed by 20 cycles of regular PCR $\left(94^{\circ} \mathrm{C}\right.$ for $30 \mathrm{sec}, 55^{\circ} \mathrm{C}$ for $30 \mathrm{sec}, 72^{\circ} \mathrm{C}$ for $\left.30 \mathrm{sec}\right)$, followed by a final extension step for $5 \mathrm{~min}$ at $72^{\circ} \mathrm{C}$. Negative controls, containing all the components except DNA templates, were included in parallel. PCR samples were stored at $-20^{\circ} \mathrm{C}$ until genotyped.

PCR amplicons were diluted with double-distilled $\mathrm{H}_{2} \mathrm{O}$ based upon their universal tail sequence (FAM and NED 
1:50, PET 1:10 and VIC 1:5) and mixed in equal amounts to provide relatively equal fluorescent signals from each locus during subsequent electrophoresis on an Applied Biosystems 3100 DNA sequencer (Applied Biosystems, Foster City, CA). Size polymorphisms were subsequently analyzed and scored using GeneScan and Genotyper software (Applied Biosystems, Foster City, CA).

\section{MLVA PCR and genotype analysis}

Primers for 32 polymorphic VNTR loci were redesigned with fluorescently labeled forward primers, and optimized for 11 multiplex PCR reactions across $B$. mallei ATCC 10399 and the 14 globally diverse B. pseudomallei isolates used in the initial screening panel (Table 4). These isolates were chosen to increase future amplification success across an array of genetically diverse isolates. MLVA reaction primers (Table 2) were designed to provide uniquely labeled or sized amplicons for every allele at all 32 VNTR loci. PCR amplification of all loci was routinely accomplished using 11 reactions, which were pooled into nine electrophoretic runs.

All reactions contained a final concentration of $1 \times$ PCR buffer, $2 \mathrm{mM} \mathrm{MgCl}_{2}, 200 \mu \mathrm{M}$ of deoxynucleoside triphosphates, 0.08 units Taq DNA Polymerase (Invitrogen, Carlsbad, CA), 1.2 M Betaine (Sigma-Aldrich Co., St. Louis, MO), double-distilled $\mathrm{H}_{2} \mathrm{O}, 1 \mu \mathrm{L}$ of template DNA $(\sim 100 \mathrm{pg} / \mu \mathrm{L})$ and the appropriate primer concentrations for each multiplex PCR (Table 1) for a total volume of 10 $\mu \mathrm{L}$. Thirteen VNTR loci required the inclusion of a nonfluorescently labeled forward primer in order to decrease the amount of fluorescent amplicon, and thus obtain relatively equal fluorescent signals from each amplicon in the multiplex mix (Table 2). In cases where low DNA quantity affected multiplex PCR results, loci were amplified individually using the same concentrations above and $0.2 \mu \mathrm{M}$ of both forward and reverse primers.

All PCR reactions were performed in MJ Research 96-well DNA engines equipped with hot bonnets (Bio-Rad, Waltham, MA). PCR reactions underwent an initial denaturation at $94^{\circ} \mathrm{C}$ for $5 \mathrm{~min}, 35$ cycles of PCR were performed (denaturation at $94^{\circ} \mathrm{C}$ for $30 \mathrm{sec}$, annealing for 30 sec at $68^{\circ} \mathrm{C}$, and an extension at $72^{\circ} \mathrm{C}$ for $30 \mathrm{sec}$ ) followed by a final extension step for $5 \mathrm{~min}$ at $72^{\circ} \mathrm{C}$. Negative controls, containing all the components except DNA templates, were included in parallel. PCR samples were stored at $-20^{\circ} \mathrm{C}$ until genotyped.

PCR products for all multiplex mixes were diluted 1:100 with double-distilled $\mathrm{H}_{2} \mathrm{O}$ and then mixed $1: 1$ with a $3: 1$ ratio of formamide to NAU Liz 1007 fluorescently labeled size standard. The PCR products were electrophorectically analyzed with an Applied Biosystems 3730xl DNA sequencer (Foster City, CA). Amplicons were scored using the ABI software program GeneMapper and genotyped according to predetermined allele sizes. An independent party has verified all sizes presented.

\section{Mutation rate determination}

A parallel serial passage experiment used to determine VNTR mutation rates began with a single isolated colony of the Bp9905-1902 strain $(\mathrm{T}=0)$. Bp9905-1902 was a human clinical isolate obtained from the Arizona Department of Health. This colony was dispersed in nutrient broth and then used to start 95 independent clonal lineages by streaking for single colonies on 24 quartered plates. Each lineage was then serially passed 10 times over a 10 day period by streaking a single colony from the previous passage. DNA was extracted from all $95 \mathrm{~T}=10$ lineages by using an in-house phenol chloroform extraction protocol. PCR for each locus was performed using the universal tail VNTR screening method described above. Mutational events were then visualized using GeneMapper software (Applied Biosystems, Foster City, CA). Using viable plate counts, the number of generations (doublings) per colony was determined to be $\sim 19.93\left(\log _{2}\right.$ of the average colony size in cells), which corresponded to a total of $1.81 \times 10^{4}$ generations in the entire experiment $(19.93$ generations/colony $\times 10$ passages $\times 91.03$ average analyzed lineages/marker), allowing the detection of mutation rates of $10^{-4}$ or greater (Table 1 ). For estimation of cell doubling see discussion and supplemental information in Girard and Wagner et al. [26,24].

\section{Statistical analyses}

Data from 23 loci that displayed greater than 85\% amplification success were used to generate an arbitrarily rooted distance-based phylogenetic tree using the Neighbor Joining algorithm in PAUP 4.0 b10 [55]. In order to estimate confidence levels for the analysis, a full heuristic bootstrapping analysis was conducted using a random generator seed for 2000 replicates. Individual marker diversity (D) was calculated as equal to $1-\sum$ (allele frequency) ${ }^{2}$ and based solely upon allele frequencies in the 87 isolates shown here (Table 1 ). A $\chi^{2}$ goodness-of-fit test was performed in $10 \mathrm{~Kb}$ intervals in order to examine the observed distribution against an expected Poisson distribution for both the large and small chromosomes.

\section{Authors' contributions}

JMU participated in the experimental design, carried out the acquisition of molecular genetic data, data analyses and interpretation and drafted the manuscript. JMS conceived the study and experimental design, carried out the genome survey including the computational and statistical analyses and drafted the manuscript. TP participated in experimental design and edited the manuscript. HH generated the molecular genetic data and edited the manuscript. KLS carried out the serial passage experiment. 
CLCF, RRLD, SDR, BL, SG, MC and LYH generated the molecular genetic data. DD, SPH, RR, DG and MM carried out the genomic DNA preparation. BJC provided epidemiological and clinical data. DW and PK obtained the funding, conceived the study, helped participate in its design and helped to draft and edit the manuscript. All authors read and approved the final manuscript.

\section{Acknowledgements}

We would like to acknowledge Dr. Amy Vogler for her assistance with the statistical analyses, as well as, Dr. Matthew Van Ert and Mr. Eric Greene for their help in the editing this manuscript. Funding for this work was provided by the U.S. Departments of Energy and Homeland Security.

\section{References}

I. White NJ: Melioidosis. Lancet 2003, 36 I(9370): I7I5-I722.

2. Rotz LD, Khan AS, Lillibridge SR, Ostroff SM, Hughes JM: Public health assessment of potential biological terrorism agents. Emerg Infect Dis 2002, 8(2):225-230.

3. Rogul M, Brendle JJ, Haapala DK, Alexander AD: Nucleic acid similarities among Pseudomonas pseudomallei, Pseudomonas multivorans, and Actinobacillus mallei. J Bacteriol 1970, I 0 I(3):827-835.

4. Holden MT, Titball RW, Peacock SJ, Cerdeno-Tarraga AM, Atkins T, Crossman LC, Pitt T, Churcher C, Mungall K, Bentley SD, Sebaihia M, Thomson NR, Bason N, Beacham IR, Brooks K, Brown KA, Brown NF, Challis GL, Cherevach I, Chillingworth T, Cronin A, Crossett B, Davis P, DeShazer D, Feltwell T, Fraser A, Hance Z, Hauser H, Holroyd S, Jagels K, Keith KE, Maddison M, Moule S, Price C, Quail MA, Rabbinowitsch E, Rutherford K, Sanders M, Simmonds M, Songsivilai S, Stevens K, Tumapa S, Vesaratchavest M, Whitehead S, Yeats C, Barrell BG, Oyston PC, Parkhill ]: Genomic plasticity of the causative agent of melioidosis, Burkholderia pseudomallei. Proc Natl Acad Sci U S A 2004, I 0 I(39): I 4240-I 4245.

5. Nierman WC, DeShazer D, Kim HS, Tettelin H, Nelson KE, Feldblyum T, Ulrich RL, Ronning CM, Brinkac LM, Daugherty SC, Davidsen TD, Deboy RT, Dimitrov G, Dodson RJ, Durkin AS, Gwinn ML, Haft DH, Khouri H, Kolonay JF, Madupu R, Mohammoud Y, Nelson WC, Radune D, Romero CM, Sarria S, Selengut J, Shamblin C, Sullivan SA, White O, Yu Y, Zafar N, Zhou L, Fraser CM: Structural flexibility in the Burkholderia mallei genome. Proc Natl Acad Sci U S A 2004, 10 I(39): 14246- I 425I.

6. Godoy D, Randle G, Simpson Al, Aanensen DM, Pitt TL, Kinoshita R, Spratt BG: Multilocus sequence typing and evolutionary relationships among the causative agents of melioidosis and glanders, Burkholderia pseudomallei and Burkholderia mallei. J Clin Microbiol 2003, 4 I (5):2068-2079.

7. Kim HS, Schell MA, Yu Y, Ulrich RL, Sarria SH, Nierman WC, DeShazer D: Bacterial genome adaptation to niches: divergence of the potential virulence genes in three Burkholderia species of different survival strategies. BMC Genomics 2005, 6: 174

8. Howe C: Glanders. In The Oxford Medicine New York, Oxford Univ. Press; 1950:185-202.

9. Srinivasan A, Kraus CN, DeShazer D, Becker PM, Dick JD, Spacek L, Bartlett JG, Byrne WR, Thomas DL: Glanders in a military research microbiologist. N Engl J Med 200I, 345(4):256-258.

10. Wheelis M: First shots fired in biological warfare. Nature 1998 , 395(6699): 213 .

11. United States. Army Medical Dept.: Military Medical Ethics Volume 2... U.S. Department Of Defense... 2003. [S.I. , s.n.; 2004.

12. Gal D, Mayo M, Smith-Vaughan H, Dasari P, McKinnon M, Jacups SP, Urquhart Al, Hassell M, Currie BJ: Contamination of hand wash detergent linked to occupationally acquired melioidosis. Am J Trop Med Hyg 2004, 7 I (3):360-362.

13. Cheng AC, Jacups SP, Gal D, Mayo M, Currie B]: Extreme weather events and environmental contamination are associated with case-clusters of melioidosis in the Northern Territory of Australia. Int J Epidemiol 2006, 35(2):323-329.

14. Sermswan RW, Wongratanacheewin S, Trakulsomboon S, Thamlikitkul V: Ribotyping of Burkholderia pseudomallei from clinical and soil isolates in Thailand. Acta Trop 200I, 80(3):237-244.
15. Currie B, Smith-Vaughan H, Golledge C, Buller N, Sriprakash KS, Kemp DJ: Pseudomonas pseudomallei isolates collected over $\mathbf{2 5}$ years from a non-tropical endemic focus show clonality on the basis of ribotyping. Epidemiol Infect 1994, I I3(2):307-3 I 2

16. Popovic T, Kim C, Reiss J, Reeves M, Nakao H, Golaz A: Use of molecular subtyping to document long-term persistence of Corynebacterium diphtheriae in South Dakota. J Clin Microbiol 1999, 37(4): 1092-1099.

17. Miettinen MK, Siitonen A, Heiskanen P, Haajanen H, Bjorkroth KJ, Korkeala HJ: Molecular epidemiology of an outbreak of febrile gastroenteritis caused by Listeria monocytogenes in coldsmoked rainbow trout. J Clin Microbiol 1999, 37(7):2358-2360.

18. Keim P, Price LB, Klevytska AM, Smith KL, Schupp JM, Okinaka R, Jackson PJ, Hugh-Jones ME: Multiple-locus variable-number tandem repeat analysis reveals genetic relationships within Bacillus anthracis. J Bacteriol 2000, I 82(10):2928-2936.

19. Haase A, Melder A, Smith-Vaughan H, Kemp D, Currie B: RAPD analysis of isolates of Burkholderia pseudomallei from patients with recurrent melioidosis. Epidemiol Infect 1995, II5(I):II5-I2I.

20. Haase A, Smith-Vaughan H, Melder A, Wood Y, Janmaat A, Gilfedder J, Kemp D, Currie B: Subdivision of Burkholderia pseudomallei ribotypes into multiple types by random amplified polymorphic DNA analysis provides new insights into epidemiology. J Clin Microbiol 1995, 33(7): 1687-1690.

21. Maiden MC, Bygraves JA, Feil E, Morelli G, Russell JE, Urwin R, Zhang Q, Zhou J, Zurth K, Caugant DA, Feavers IM, Achtman M, Spratt BG: Multilocus sequence typing: a portable approach to the identification of clones within populations of pathogenic microorganisms. Proc Natl Acad Sci U S A 1998, 95(6):3 I40-3। 45.

22. Liu Y, Loh JP, Aw LT, Yap EP, Lee MA, Ooi EE: Rapid molecular typing of Burkholderia pseudomallei, isolated in an outbreak of melioidosis in Singapore in 2004, based on variablenumber tandem repeats. Trans $R$ Soc Trop Med Hyg 2006, 100(7):687-692.

23. Johansson $A$, Farlow J, Larsson $P$, Dukerich $M$, Chambers $E$, Bystrom M, Fox J, Chu M, Forsman M, Sjostedt A, Keim P: Worldwide genetic relationships among Francisella tularensis isolates determined by multiple-locus variable-number tandem repeat analysis. J Bacteriol 2004, I 86( I 7):5808-58I8.

24. Le Flèche $P$, Hauck $Y$, Onteniente $L$, Prieur $A$, Denoeud $F$, Ramisse $V$, Sylvestre $P$, Benson G, Ramisse F, Vergnaud G: A tandem repeats database for bacterial genomes: application to the genotyping of Yersinia pestis and Bacillus anthracis. BMC Microbiology 200I, I(I):2.

25. Suraka S, Toman R, Skultety L, Slaba K, Homan WL: Establishment of a genotyping scheme for Coxiella burnetii. FEMS Microbiol Lett 2006, 254(2):268-274.

26. Girard JM, Wagner DM, Vogler AJ, Keys C, Allender CJ, Drickamer LC, Keim P: Differential plague-transmission dynamics determine Yersinia pestis population genetic structure on local, regional, and global scales. Proc Natl Acad Sci U S A 2004, $101(22): 8408-8413$.

27. Hoffmaster AR, Fitzgerald CC, Ribot E, Mayer LW, Popovic T: Molecular subtyping of Bacillus anthracis and the $200 \mathrm{I}$ bioterrorism-associated anthrax outbreak, United States. Emerg Infect Dis 2002, 8(10): IIII-III6.

28. Budowle B, Johnson MD, Fraser CM, Leighton TJ, Murch RS, Chakraborty R: Genetic analysis and attribution of microbial forensics evidence. Crit Rev Microbiol 2005, 3 I (4):233-254.

29. Johansson A, Forsman M, Sjostedt A: The development of tools for diagnosis of tularemia and typing of Francisella tularensis. Apmis 2004, I I 2(I I- I 2):898-907.

30. Lindstedt BA: Multiple-locus variable number tandem repeats analysis for genetic fingerprinting of pathogenic bacteria. Electrophoresis 2005, 26(1 3):2567-2582.

3I. Burkholderia pseudomallei Multi Locus Sequencing Typing Database [http://bpseudomallei.mlst.net/]

32. Harvey SP, Minter JM: Ribotyping of Burkholderia mallei isolates. FEMS Immunol Med Microbiol 2005, 44(I):91-97.

33. Cheng AC, Currie BJ: Melioidosis: epidemiology, pathophysiology, and management. Clin Microbiol Rev 2005, I 8(2):383-4I6.

34. Levy A, Chang BJ, Abbott LK, Kuo J, Harnett G, Inglis TJ: Invasion of spores of the arbuscular mycorrhizal fungus Gigaspora decipiens by Burkholderia spp. Appl Environ Microbiol 2003, 69(10):6250-6256. 
35. Inglis TJ, Rigby P, Robertson TA, Dutton NS, Henderson M, Chang B: Interaction between Burkholderia pseudomallei and Acanthamoeba species results in coiling phagocytosis, endamebic bacterial survival, and escape. Infect Immun 2000, 68(3): $1681-1686$

36. Wuthiekanun V, Smith MD, Dance DA, White NJ: Isolation of Pseudomonas pseudomallei from soil in north-eastern Thailand. Trans R Soc Trop Med Hyg 1995, 89(1):4I-43.

37. Howard K, Inglis T]: Novel selective medium for isolation of Burkholderia pseudomallei. J Clin Microbiol 2003, $4 I(7): 33 \mid 2-3316$.

38. Dawid S, Barenkamp SJ, St Geme JW 3rd: Variation in expression of the Haemophilus influenzae HMW adhesins: a prokaryotic system reminiscent of eukaryotes. Proc Natl Acad Sci U S A 1999, 96(3): 1077-1082

39. van Ham SM, van Alphen L, Mooi FR, van Putten JP: Phase variation of $H$. influenzae fimbriae: transcriptional control of two divergent genes through a variable combined promoter region. Cell 1993, 73(6): I I87-II 196.

40. van Belkum A, van Leeuwen W, Scherer S, Verbrugh H: Occurrence and structure-function relationship of pentameric short sequence repeats in microbial genomes. Res Microbiol 1999, I 50(9-10):617-626

4l. van Belkum A: Short sequence repeats in microbial pathogenesis and evolution. Cell Mol Life Sci 1999, 56(9-10):729-734.

42. Romero CM, DeShazer D, Feldblyum T, Ravel J, Woods D, Kim HS, Yu Y, Ronning CM, Nierman WC: Genome sequence alterations detected upon passage of Burkholderia mallei ATCC 23344 in culture and in mammalian hosts. BMC Genomics 2006, 7:228.

43. Vogler AJ, Keys C, Nemoto Y, Colman RE, Jay Z, Keim P: Effect of repeat copy number on variable-number tandem repeat mutations in Escherichia coli O157:H7. J Bacteriol 2006, I 88( ( 2):4253-4263.

44. Lovett ST: Encoded errors: mutations and rearrangements mediated by misalignment at repetitive DNA sequences. Mol Microbiol 2004, 52(5): 1243-1253.

45. Mrazek J: Analysis of distribution indicates diverse functions of simple sequence repeats in Mycoplasma genomes. Mol Biol Evol 2006, 23(7): I370-। 385.

46. Zivanovic $\mathrm{Y}$, Lopez P, Philippe H, Forterre P: Pyrococcus genome comparison evidences chromosome shuffling-driven evolution. Nucleic Acids Res 2002, 30(9): 1902-1910.

47. Watt VM, Ingles CJ, Urdea MS, Rutter WJ: Homology requirements for recombination in Escherichia coli. Proc Natl Acad Sci U S A 1985, 82(14):4768-4772

48. Vogler AJ Keys, CE, Allender, C, Bailey, I, Girard, J, Pearson,T,, Smith KL Wagner, DM, and Keim, P: Mutations, mutation rates, and evolution at the hypervariable VNTR loci of Yersinia pestis. Mutation Research 2007, 616 ( I-2 ): 145-I58.

49. Pearson T, U'Ren JM, Schupp JM, Allan GJ, Foster PG, Mayo MJ, Gal $D$, Choy JL, Leadem Daugherty R, Kachur S, Clark Friedman CL, Leadem B, Georgia S, Hornstra H, Vogler AJ, Wagner DM, Keim P, Currie B]: VNTR analysis of selected outbreaks of Burkholderia pseudomallei in Australia. Infect Genet Evol 2006 in press.

50. Vesaratchavest M, Tumapa S, Day NP, Wuthiekanun V, Chierakul W, Holden MT, White NJ, Currie BJ, Spratt BG, Feil EJ, Peacock SJ: Nonrandom Distribution of Burkholderia pseudomallei Clones in Relation to Geographical Location and Virulence. J Clin Microbiol 2006, 44(7):2553-2557.

5I. McCombie RL, Finkelstein RA, Woods DE: Multilocus sequence typing of historical Burkholderia pseudomallei isolates collected in Southeast Asia from 1964 to 1967 provides insight into the epidemiology of melioidosis. J Clin Microbiol 2006, 44(8):295I-2962

52. Cheng AC, Godoy D, Mayo M, Gal D, Spratt BG, Currie BJ: Isolates of Burkholderia pseudomallei from Northern Australia are distinct by multilocus sequence typing, but strain types do not correlate with clinical presentation. J Clin Microbiol 2004, 42( I 2):5477-5483.

53. Benson G: Tandem repeats finder: a program to analyze DNA sequences. Nucleic Acids Res 1999, 27(2):573-580.

54. Jay Z: Discovery and analysis of Bacillus anthracis chromosomal VNTRs using universal primers. In Department of Biological Sciences Volume M.S.. Flagstaff, Northern Arizona University; 2005.
55. Swofford DL: PAUP, Phylogenetic Analysis Using Parsimony (and Other Methods), Version 4.0 Beta. Sunderland, MA, Sinauer; 1999.
Publish with Bio Med Central and every scientist can read your work free of charge

"BioMed Central will be the most significant development for disseminating the results of biomedical research in our lifetime. "

Sir Paul Nurse, Cancer Research UK

Your research papers will be:

- available free of charge to the entire biomedical community

- peer reviewed and published immediately upon acceptance

- cited in PubMed and archived on PubMed Central

- yours - you keep the copyright 